\title{
Isolation and preparation of naturally occurring aluminum ligands using immobilized metal affinity chromatography for analysis by electrospray ionization-mass spectrometry
}

\author{
Carson Baldwin \\ West Virginia University
}

Follow this and additional works at: https://researchrepository.wvu.edu/etd

\footnotetext{
Recommended Citation

Baldwin, Carson, "Isolation and preparation of naturally occurring aluminum ligands using immobilized metal affinity chromatography for analysis by electrospray ionization-mass spectrometry" (2005). Graduate Theses, Dissertations, and Problem Reports. 2210.

https://researchrepository.wvu.edu/etd/2210
}

This Thesis is protected by copyright and/or related rights. It has been brought to you by the The Research Repository @ WVU with permission from the rights-holder(s). You are free to use this Thesis in any way that is permitted by the copyright and related rights legislation that applies to your use. For other uses you must obtain permission from the rights-holder(s) directly, unless additional rights are indicated by a Creative Commons license in the record and/ or on the work itself. This Thesis has been accepted for inclusion in WVU Graduate Theses, Dissertations, and Problem Reports collection by an authorized administrator of The Research Repository @ WVU. For more information, please contact researchrepository@mail.wvu.edu. 
ISOLATION AND PREPARATION OF NATURALLY OCCURRING ALUMINUM LIGANDS USING IMMOBILIZED METAL AFFINITY CHROMATOGRAPHY FOR ANALYSIS BY ELECTROSPRAY IONIZATION-MASS SPECTROMETRY

\author{
by \\ Carson Baldwin \\ Thesis submitted to the Eberly College of Arts and Sciences \\ at West Virginia University \\ in partial fulfillment of the requirements \\ for the degree of \\ Master of Science \\ in \\ Chemistry \\ Approved by \\ Aaron T. Timperman, Ph.D., Chair \\ Fred L. King, Ph.D. \\ Ronald B. Smart, Ph.D. \\ C. Eugene Bennett Department of Chemistry \\ Morgantown, West Virginia \\ 2005
}

Keywords: Aluminum, IMAC, isolation, chromatography, ligand, mass spectrometry

Copyright 2005 Carson Baldwin 


\section{Abstract \\ ISOLATION AND PREPARATION OF NATURALLY OCCURRING ALUMINUM LIGANDS USING IMMOBILIZED METAL AFFINITY CHROMATOGRAPHY FOR ANALYSIS BY ELECTROSPRAY IONIZATION-MASS SPECTROMETRY}

\section{Carson Baldwin}

Typically, $\mathrm{Al}^{3+}$ is bound to minerals, but its toxicity towards vascular plants increases with increasing soil acidity. Ectomycorrhizal fungi often confer $\mathrm{Al}^{3+}$ resistance to host plants by unknown mechanisms. The objective of this research was to develop a method for the successful separation of $\mathrm{Al}^{3+}$ binding fungal exudates produced by the fungus Pisolithus tinctorius. A novel $\mathrm{Al}^{3+}$ immobilized metal affinity chromatography (IMAC) technique was developed to extract exudates involved in $\mathrm{Al}^{3+}$ binding. Recovery of exudates from the IMAC column was dependent on elutor type and $\mathrm{pH}$, with ammonium hydroxide being most effective. Exudates exhibited strong binding with $\mathrm{Al}^{3+}$, allowing their selective enrichment and collection. Because IMAC strongly binds $\mathrm{Al}^{3+}$ that forms an $\mathrm{Al}^{3+}$-ligand complex, isolation of $\mathrm{Al}^{3+}$ ligands was possible. Exudates are also separated using reversed phase in order to differentiate between closely related compounds. Utilizing these techniques results in a simpler sample to be characterized with mass spectrometry. 


\section{ACKNOWLEDGMENTS}

I'd like to give a deep thank you to my advisor Dr. Aaron Timperman as well as my collaborator Dr. Jonathan Cumming. Thanks to both of them, I have gained the experience and knowledge to be a successful scientist. They taught me the necessary skills to be a responsible and effective chemist and adapt to any task needed.

I also owe thanks to my committee members Dr. Fred King and Dr. Ronald Smart for their assistance through the course of my studies. Furthermore, I thank my lab coworkers whom have all lent a hand at some point along the way. A special thank you to Jim Lenke whose knowledge and desire to aid me helped support me during my research.

Finally, I thank my family and friends, especially my mother and father, who supported and motivated me, not only through my time at West Virginia University, but throughout my life. Without them, I would not be who or where I am now. 


\section{TABLE OF CONTENTS}

Title

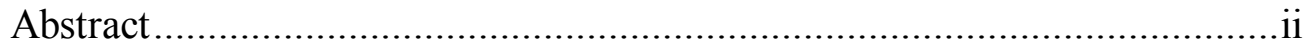

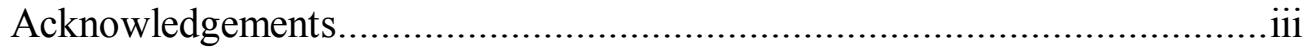

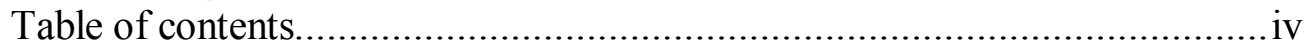

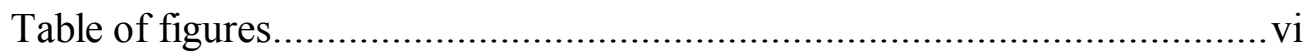

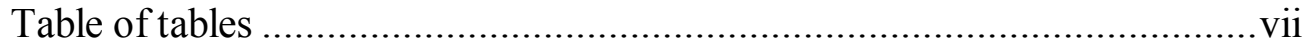

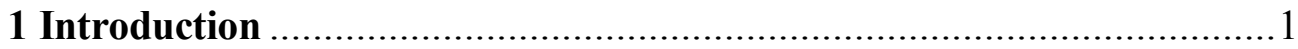

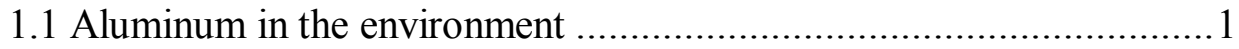

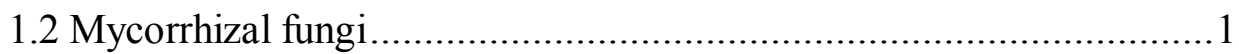

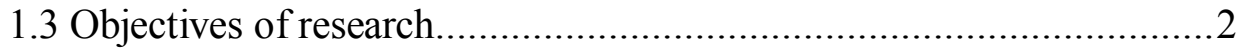

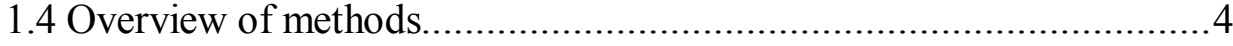

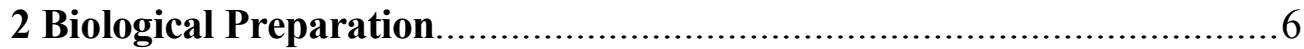

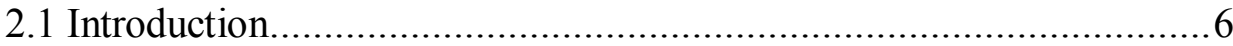

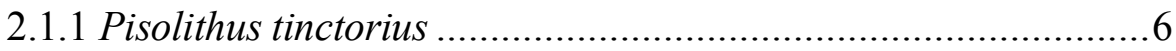

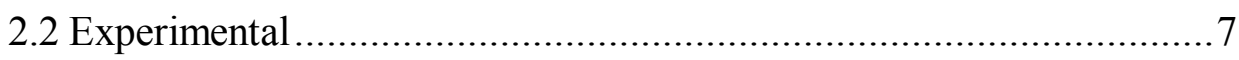

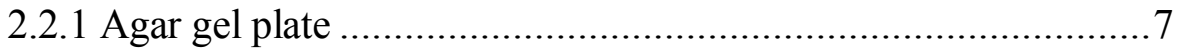

2.2.2 Growing fungus ...............................................................

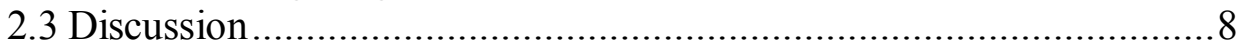

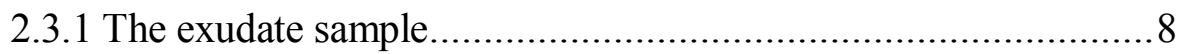

3 Immobilized Metal Affinity Chromatography.................................... 10

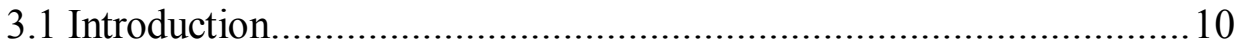

3.1.1 Principle of IMAC …….................................................... 10

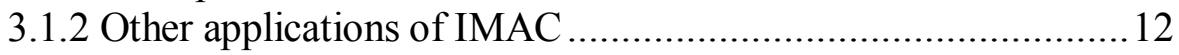

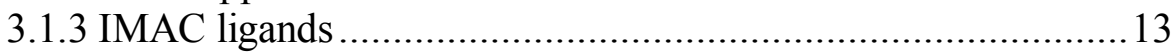

3.1.4 Hard metal IMAC .........................................................

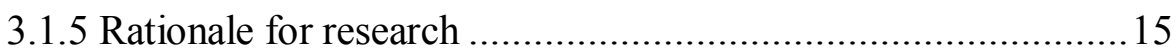

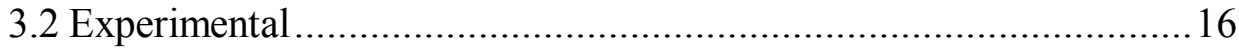

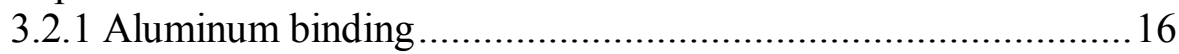

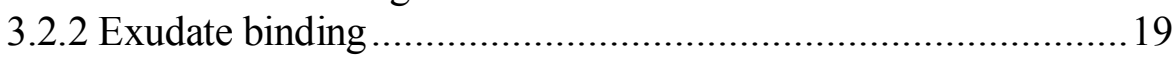

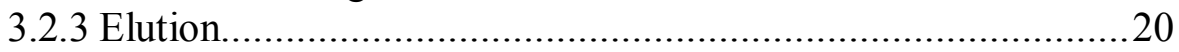

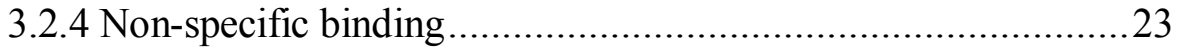

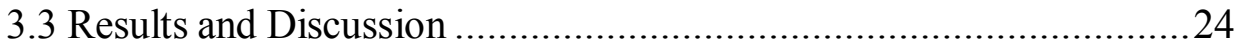

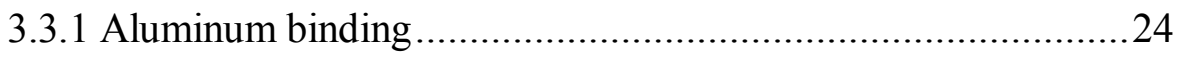

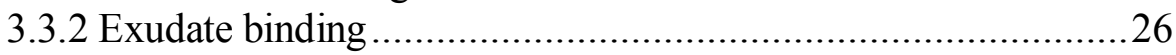

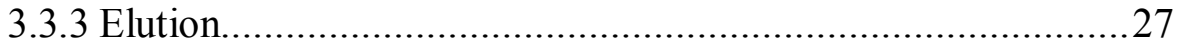

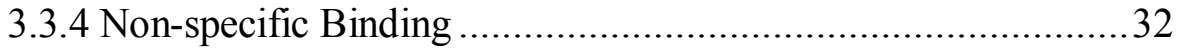

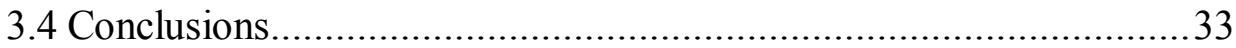

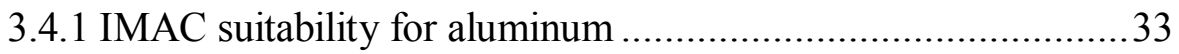

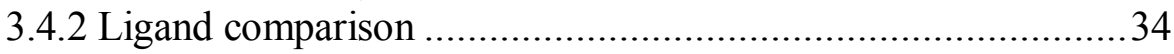

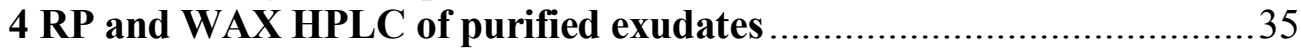

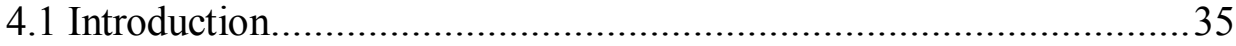

4.1.1 Principle of reversed phase separation ........................................ 35 
4.1.2 Principle of weak anion exchange separation .........................35

4.1.3 Coupling of WAX and RP................................................36

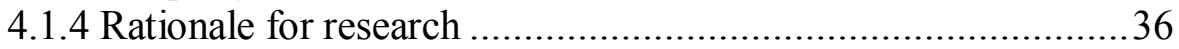

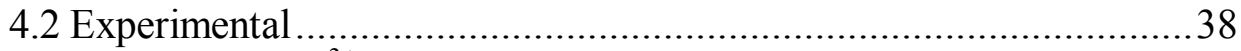

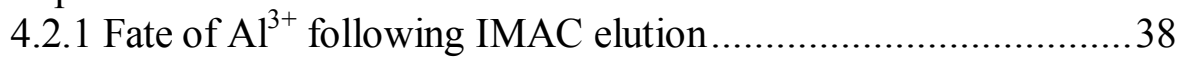

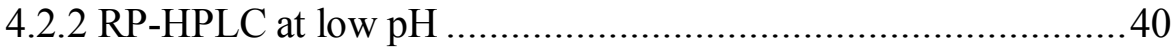

4.2.3 RP-HPLC at high $\mathrm{pH}$...................................................... 40

4.2.4 WAX of unbound exudate.................................................. 42

4.3 Results and Discussion .......................................................... 43

4.3.1 Fate of $\mathrm{Al}^{3+}$ following IMAC elution..................................43

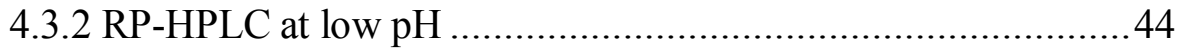

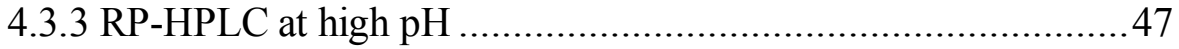

4.3.4 WAX of unbound exudate..................................................50

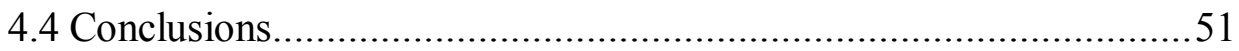

4.4.1 Advantage of multiple separations .......................................51

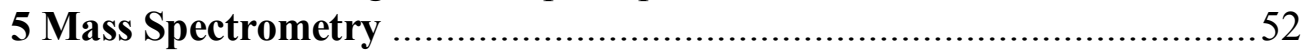

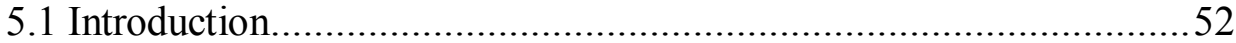

5.1.1 Principle of ESI-MS ......................................................52

5.1.2 Rationale for Research ...................................................55

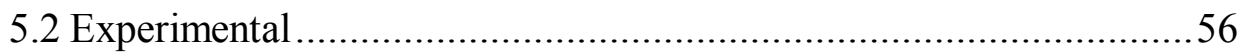

5.2.1 Direct infusion of exudate ...............................................56

5.2.2 Removal of free $\mathrm{Al}^{3+}$ from the IMAC eluent using RP extraction

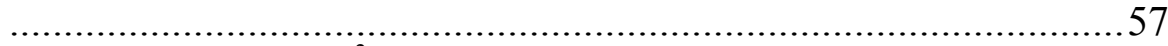

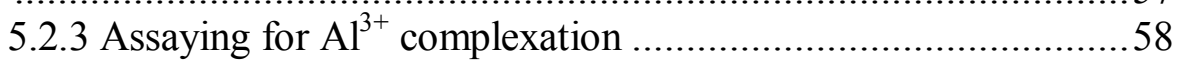

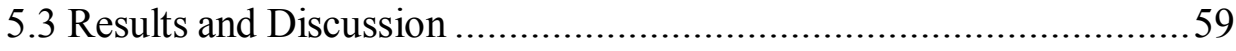

5.3.1 Direct infusion of exudate ...............................................59

5.3.2 Removal of free $\mathrm{Al}^{3+}$ from the IMAC eluent using RP extraction

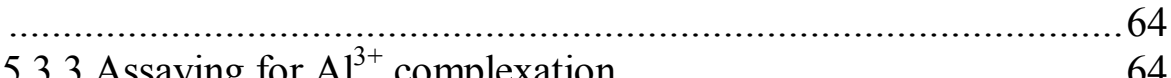

5.4 Conclusions.............................................................. 70

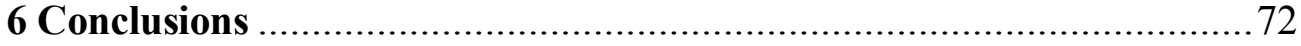

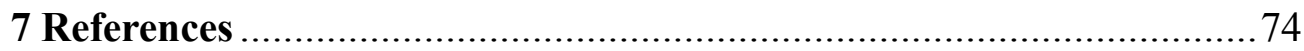




\section{TABLE OF FIGURES}

Figure 1.1-Path of fungal exudates through research ................................. 3

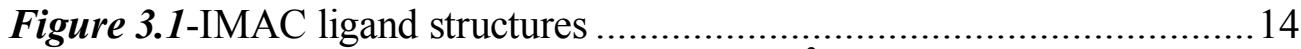

Figure 3.2-Exudate binding with and without $\mathrm{Al}^{3+}$ loaded ...........................26

Figure 3.3-IMAC elution as a function of $\mathrm{pH}$..........................................28

Figure 3.4A-Exudate absorbance shift with oxalic acid present .................. 31

Figure 3.4B-Exudate absorbance shift with tiron present ........................ 31

Figure 3.4C-Exudate absorbance shift with $\mathrm{NH}_{4} \mathrm{OH}$ present ...................... 32

Figure 3.5-Exudate \& ammonium hydroxide absorbance......................... 32

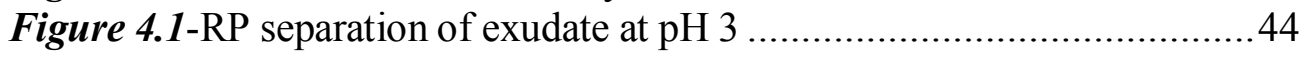

Figure 4.2-RP of IMAC fraction at $\mathrm{pH} 3$............................................ 45

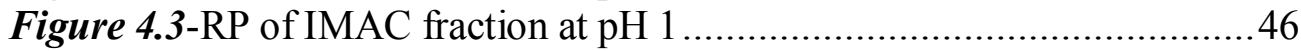

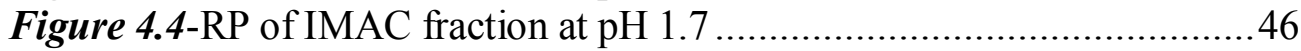

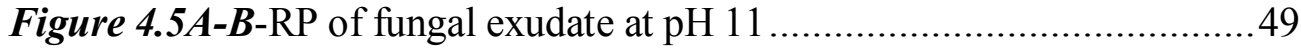

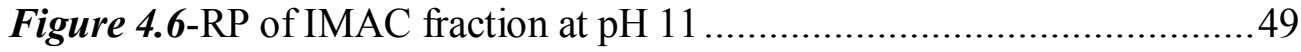

Figure 5.1-Schematic of ESI-MS ....................................................... 52

Figure 5.2-MS spectrum of fungal exudate in positive ion mode................6 61

Figure 5.3-MS spectrum of fungal exudate in negative ion mode................62

Figure 5.4-MS spectrum of IMAC isolated exudate in positive ion mode.....63

Figure 5.5-MS spectrum of IMAC isolated exudate in negative ion mode....63

Figure 5.6-Mass spectrum of post IMAC-RP sample .............................6 68

Figure 5.7-Mass spectrum of post IMAC-RP sample................................68

Figure 5.8-Mass spectrum of post IMAC-RP sample...............................6 69

Figure 5.9-Mass spectrum of post IMAC-RP sample...............................69

Figure 5.10-Mass spectrum of background solution............................... 70 


\section{TABLE OF TABLES}

Table 3.1-Experimental conditions for $\mathrm{Al}^{3+}$ binding on IMAC ...................18

Table 3.2-Stability constants for NTA with different metals ......................25

Table 3.3-IMAC model compound comparison .....................................2 27

Table 3.4-IMAC elutor efficiency ..........................................................28

Table 4.1-Fate of $\mathrm{Al}^{3+}$ following RP purification ....................................50 


\section{Introduction}

\subsection{Aluminum in the environment}

Aluminum $\left(\mathrm{Al}^{3+}\right)$ is a major component of most soils, where it plays a structural role in clays and amorphous minerals. Aluminum exhibits $\mathrm{pH}$-dependent solubility and speciation, and, under acidic conditions, the $\mathrm{Al}^{3+}$ may dominate soil chemistry [1]. Once in solution, $\mathrm{Al}^{3+}$ is phytotoxic, inhibiting root cell division and growth, altering water and ion acquisition, and, ultimately, the growth of plants on acidic soils [2, 3]. Worldwide, acidic soil is an impediment to crop production on 30 to $40 \%$ of arable lands, second only to drought as a limiting factor [4].

However, many crop and forest species exhibit some resistance to $\mathrm{Al}^{3+}$ in soils. Aluminum resistance in many plant species is associated with plant-altered rhizosphere chemistry and $\mathrm{Al}^{3+}$ speciation through the production of low molecular weight organic acids $[2,3,5,6]$.

\subsection{Mycorrhizal fungi}

In addition to $\mathrm{Al}^{3+}$-binding ligands produced by plants, the exudation of organic metal-chelating compounds by symbiotic mycorrhizal fungi may additionally alter $\mathrm{Al}^{3+}$ speciation in soils and subsequent toxicity to plants. Mycorrhizal fungi form symbiotic associations with the roots of crop and forest tree species, and play important roles in nutrient and water uptake $[7,8]$. Both ectomycorrhizal fungi and arbuscular mycorrhizal fungi have been shown to produce compounds that have the potential to chelate $\mathrm{Al}^{3+}[9$, 10]. As these fungi naturally form symbioses with the majority of vascular plants, a clearer understanding of the types of potential metal-chelating compounds produced by mycorrhizal fungi will elucidate possible mechanisms of metal resistance in higher plants. 
Root and fungal exudates are complex mixtures containing a variety of potential metalbinding compounds.

Elevated levels of $\mathrm{Al}^{3+}$ and other metal ions in acidic soils pose challenges for plant growth in many regions of the world $[1,4]$. Many plants show some degree of resistance to metal ions and interactions between plant and fungi are known to be important. Some mechanisms of metal resistance have been determined, but they cannot fully account for the observed extent of metal binding. Small organic acids, particularly citrate, malate, and oxalate are known to bind $\mathrm{Al}^{3+}$, reducing its bioavailability. However, these small organic acids cannot account for the total metal binding ligands in the soil and it is our hypothesis that the fungal pigment exudates also play an important role in metal binding. We have used the ectomycorrhizal fungus Pisolithus tinctorius as a model organism. P. tinctorius has served as a model ectomycorrhizal system for numerous years. This fungus was first isolated by D.H. Marx in 1970 from coal mine spoil in Ohio and has proved to be a strong colonizer of pine and aided in the reforestation of abandoned coal mines [30]. Subsequent work since then has implicated roles for P. tinctorius in aluminum, copper, nickel, zinc, and manganese resistance [31]. Its pigment exudates are a complex mixture of compounds that are yellow-brown in color and have many properties similar to humic acids. In this research, immobilized metal affinity chromatography (IMAC) has been employed to extract $\mathrm{Al}^{3+}$ metal binding ligands from the exudate mixture.

\subsection{Objectives of research}

The first objective of this research was to selectively extract the $\mathrm{Al}^{3+}$ binding ligands from the complex sample mixture using $\mathrm{Al}^{3+} \mathrm{IMAC}$. The exudate mixture contains several sugars, vitamins, and other exudates and fungal materials that do not bind $\mathrm{Al}^{3+}$ that 
form a complex background matrix, which must be removed before analysis. Secondly, because the isolated $\mathrm{Al}^{3+}$ ligands remain in a heterogeneous mixture, further purification must follow the IMAC extraction. A requirement of the purification process is the fungal exudate sample must be compatible with electrospray ionization-mass spectrometry (ESIMS) analysis. Therefore, an important step following elution of the exudates from the IMAC cartridge is preparation of the samples using reversed phase-liquid chromatography (RP-LC) for ESI-MS analysis. The final goal of this work was to demonstrate that the sample was pure enough and the complexity adequately reduced for ESI-MS analysis. In the end, the overall development of a method for the isolation and purification of fungal exudates for downstream ESI-MS analysis was the ultimate goal of this research. Figure 1.1 shows the experimental routes taken to meet these objectives.

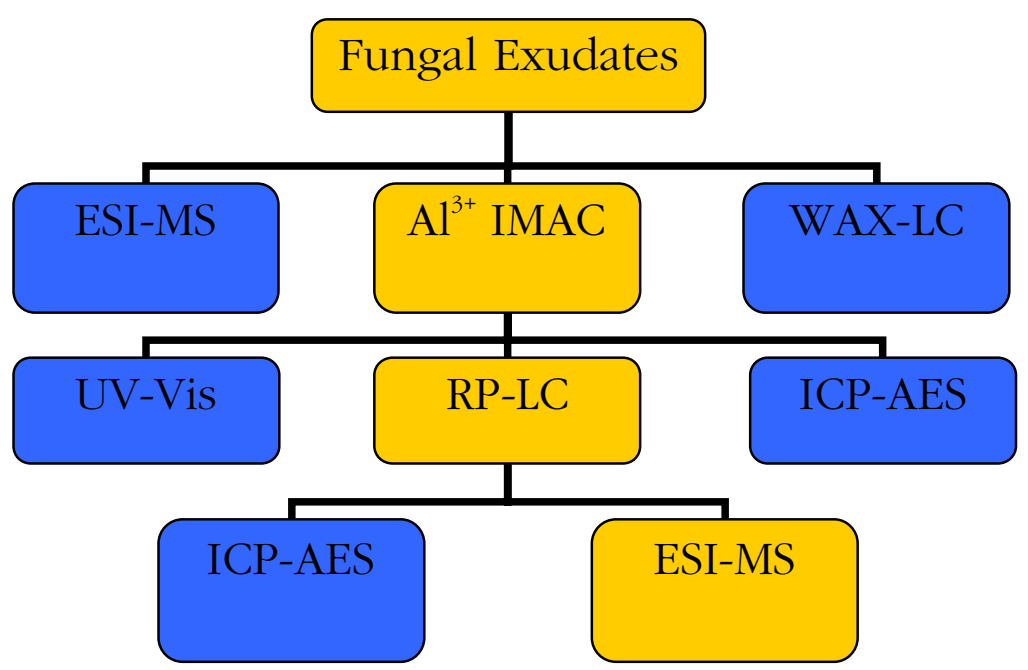

Figure 1.1-Methods used at different stages during development of fungal exudate purification. Blue boxes represent techniques used to evaluate or quantitate method. Gold boxes represent path routinely taken for final sample preparation analysis. 


\subsection{Overview of methods}

The developed method for isolation and analysis of the $\mathrm{Al}^{3+}$ binding ligands utilizes $\mathrm{Al}^{3+}$ IMAC, reversed phase extraction, and qualitative analysis by ESI-MS. Although IMAC has been used widely with metals ions such as $\mathrm{Ni}^{2+}$ and $\mathrm{Cu}^{2+}$, there are only a few previous reports using $\mathrm{Al}^{3+}$ with details regarding the metals behavior on an IMAC column $[21,22]$. IMAC has been developed into an excepted technique for the isolation of metal cations and metal binding analyte ligands. However, due to the lack of established $\mathrm{Al}^{3+}$ IMAC methodology available, optimizing a successful IMAC extraction was a core focus. Therefore, initial work centered on the development of an $\mathrm{Al}^{3+}$ IMAC method for the isolation and recovery of fungal exudates that bind $\mathrm{Al}^{3+}$.

Following $\mathrm{Al}^{3+}$ IMAC isolation, ultraviolet-visible (UV-Vis) spectroscopy and inductively coupled plasma-atomic emission spectroscopy (ICP-AES) were used for semiquantitative analysis of the IMAC isolated fractions. Using UV-Vis spectroscopy following IMAC isolation, it was possible to determine and compare the relative concentration of exudates eluted from the IMAC column. ICP-AES was used for quantitation of metal ions and to determine the binding capacity and elution behavior of the $\mathrm{Al}^{3+}$ from the IMAC column.

Due to the mechanism of elution in the IMAC isolation, further purification of the eluent was needed. Initially reversed phase high-pressure liquid chromatography (RPHPLC) was used to separate the sample mixture based on hydrophobicity. This method was chosen because the $\mathrm{Al}^{3+}$ ligands were expected to be hydrophobic; containing multiple aromatic rings and aliphatic chains. Fractions from the RP-HPLC extractions were then analyzed using ICP-AES to ascertain whether $\mathrm{Al}^{3+}$ is free in solution or complexed with 
exudates after IMAC isolation. Additionally, RP buffers are compatible with ESI-MS which was used for qualitative analysis.

ESI-MS was used to gather structural information about the purified samples. MS analysis results provide mass/charge $(\mathrm{m} / \mathrm{z})$ data that can be interpreted to reveal structural information about the molecule. IMAC isolated exudates were analyzed to observe the signal present after the removal of $\mathrm{Al}^{3+}$ from the matrix. A direct assay was performed by lowering sample $\mathrm{pH}$ and spiking $\mathrm{Al}^{3+}$ back into the sample to observe any ion signal shifts that occurred after this addition due to complexation. If spectra from these two samples match, then it may be deduced that the exudates retain $\mathrm{Al}^{3+}$ after IMAC isolation. If the spectra differ, then the exudates were uncomplexed after IMAC and rebind $\mathrm{Al}^{3+}$ in solution. 


\section{Biological preparation}

\subsection{Introduction}

Mycorrhizal fungi are very common throughout the environment, living on the roots of over $90 \%$ of vascular plants, and confer metal tolerance to their host plant. Yet despite their prevalence in soil, little is known about their mechanism of metal binding and the molecular structure of these exudates has not been investigated. Therefore, using real fungus as the source of the pigment sample is necessary in order to determine the true behavior of its components. In order to do this, fungus cultures were grown under controlled conditions to ensure as high a level of uniformity between samples as possible, thereby, increasing the experimental reproducibility of the biosynthetically produced exudates.

\subsubsection{Pisolithus tinctorius}

Mycorrhizal fungi and vascular plants exist in a symbiotic relationship wherein the fungi exist on the root systems of the plants, retrieving nutrients from the plant roots. In return, the fungi infer toxin tolerance to the host plant. The fungi possess an elaborate root system that intertwines with the roots of the plant. It is observed that plants that live in the presence of mycorrhizal fungi are healthier than plants without the large density of fungi roots around their base. This occurrence is due to the higher tolerance to toxins, such as $\mathrm{Al}^{3+}$, imparted by the presence of the fungi. Although mycorrhizal fungi are a wide category of fungi, Pisolithus tinctorius is a specific example of a mycorrhizal fungus that

serves as our model fungus. P. tinctorius is found widely across the United States and is typically located near conifers and sometimes oaks. $P$. tinctorius was chosen as the model 
organism for this study because it is widespread throughout the United States, hardy, easy to culture, and has short, ectomycorrhizal root systems.

\subsection{Experimental}

\subsubsection{Agar gel plate}

The $P$. tinctorius fungal cultures are grown on agar gels following standard procedures [10]. The agar gels provide a solid support for the fungus to grow on while providing the necessary nutrients needed for proper growth of the fungal cultures. Because agar and media are non-specific, the cultures must be prepared in a sterile environment. Before working in the hood, all surfaces must be cleaned with ethanol and the blower and UV lamp activated to sterilize all tools and plates. Agar gel consisted of modified MelinNorkans media [10] and consists of a variety of sugars and vitamins. The $\mathrm{pH}$ was then adjusted to approximately 5 using $1 \mathrm{MNaOH}$. The agar $(8.0 \mathrm{~g})$ was then added to gel the mixture and autoclaved for sterilization. The agar gel is poured into the plates and allowed to cool and solidify overnight in the hood. Once the gel has formed, the plates are ready to support the fungi during its growth period.

\subsubsection{Growing fungus}

Pisolithus tinctorius was cultured following the methods of Cumming et al. [10]. The first stage of fungal culturing is growth in the agar gel. A cork puller is used to remove uniformly sized plugs of the fungi from the pre-existing mature batch. This fungi plug is placed in the center of the fresh gel. The plate is covered with the lid and sealed with parafilm to avoid contamination during the growth period. The plates are incubated in the dark for four weeks. 
For production and recovery of the exudates, the fungus must be transferred to a liquid media. The media contains essentially the same components as the agar gel except the solidifying agar gel is not included. In doing so, the fungal exudates leach into the liquid media, resulting in a sample mixture that is used through the course of this work. For the production of fungal exudates, a series of sterilized $250 \mathrm{~mL}$ Erlenmeyer flasks containing $40 \mathrm{~mL}$ of liquid Modified Melin-Norkans media were prepared. These flasks of media were modified to optimize metal ion solubility according to method established by Cumming et al. [10]. Three plugs from the mature agar plate fungi were then added to each flask that was then loosely corked and covered with foil. These flasks were incubated for four weeks and the exudates were collected by filtering cultures sequentially through quantitative filter paper and $0.45 \mu \mathrm{m}$ membrane filters using vacuum. Sterile exudates were stored at $4^{\circ} \mathrm{C}$ in sealed $50 \mathrm{~mL}$ centrifuge tubes for subsequent analysis.

\subsection{Discussion}

\subsubsection{The exudate sample}

It is our hypothesis that the fungal exudate pigments that are being targeted are at least partially responsible for the metal binding ability of the fungi [10]. Because the exudate pigment ligands are produced biosynthetically, the sample has a number of important characteristics that should be noted. First, the sample is a complex mixture of many $\mathrm{Al}^{3+}$ binding compounds. Second, although every effort was made to maintain uniform and consistent growth conditions, it is impossible to produce exactly the same sample mixture between exudate batches. The stochastic nature of the states of the individual cells within the population prevents the reproducible production of exudates. Third, the variability is expected to be low to moderate, but the variability cannot be 
measured using the methods available. Forth, samples are observed to degrade over time by either precipitating out of solution or change in color. Due to sample degradation, the mass production and long-term storage of samples was not practical. Samples stored at $4^{0} \mathrm{C}$ resulted in precipitation over time and samples that were dried down could not be resolubilized. Ultimately, new fungal cultures had to be grown and new exudate ligands collected as needed. These considerations also led to the inability to prepare proper standards for the fungal exudates. Consequently, absorbance data and mass spectral data from different cultures may show variations and preparing a true standard was not possible. However, comparing data gathered from within the same culture batch and at relatively close periods of time should contain little or no potential error since the samples come from the same solution and have experienced the same conditions during preparation and storage. In the future, the methods developed here could be used to assess the reproducibility of each culture. The exudates' overall behavior as a metal chelator should not be expected to change. The exact structure may differ but the primary composition will remain the same. When interpreting mass spectra or atomic emission data, the reader should keep in mind the differences seen between experiments performed at different points in time result from the natural irregularity in the samples. The primary goal of this research was to develop a method suitable to characterize this class of biomolecules, not strictly identify these samples specifically. 


\section{Immobilized Metal Affinity Chromatography}

\subsection{Introduction}

\subsubsection{Principle of IMAC}

IMAC retains analytes based on analyte-metal interactions, where open binding sites on immobilized metal ions coordinate metal binding ligands in the analyte solution. IMAC has become more common place and widespread as the need for protein and peptide purification techniques has increased. The primary application of IMAC is the effective separation of proteins and peptides containing metal binding sites, such as histidine or phosphorylated sites, using immobilized intermediate metals. However, the ability of IMAC to easily separate compounds based on their metal binding is worth exploring using a wider range of metal cations. IMAC has established its place for use with intermediate metals due to their coordination chemistry and universal complexing. Due to the hard nature of $\mathrm{Al}^{3+}$, the traditional IMAC methods, such as acid or amine-containing elutors, for intermediate metals were ineffective for $\mathrm{Al}^{3+}$. Therefore, novel IMAC methods were developed to achieve the set objectives.

IMAC can utilize many types of metal binding ligands, but iminodiacetic acid (IDA) is most widely used and is commercially available [13]. The surface of the IMAC stationary phase is coated with metal binding ligands to complex and immobilize the metal ions. The metal ions are only partially complexed by the immobilizing ligand, leaving other binding sites on the metal free to interact with metal binding ligands from the sample. Elution of the analyte ligands can be achieved by: introducing a competing metal ligand, introducing free metal ions, or adjusting the $\mathrm{pH}$ [11]. Ideally, the analyte ligands are eluted as free ligands in solution and the metal ions are retained on the IMAC column. Another 
mechanism for elution is metal ion transfer by which the metal and analyte are eluted as a complex [11].

IMAC was originally developed by Jerker Porath in 1975 [23]. At first, IMAC was not a widely used technique. As the popularity of studying peptides and proteins grew, so did the applicability of IMAC. The increased use of IMAC was largely due to its application to the selective enrichment or separation of certain proteins and peptides. IMAC can be used specifically for phosphopeptides, histidine containing proteins, and other metal specific compounds, a functionality which had not previously been available [11]. Originally IMAC methods used intermediate metal ions such as $\mathrm{Ni}^{2+}, \mathrm{Cu}^{2+}$, or $\mathrm{Zn}^{2+}$ [22]. Intermediate metals, or those whose properties fall in the intermediate portion of the relative hard/soft acid/base scale, are advantageous because they bind to the imidazole or thiol sites in peptides [22]. However, IMAC resins containing IDA ligands still bind intermediate metals strong enough so that the peptide is eluted from the column while the metal remains immobilized on the column using the desorption elution mechanism [11]. IDA is the most common ligand used in IMAC, although with an increasing number of IMAC applications, a growing number of ligands are being developed, such as nitrilotriacetic acid (NTA) and immobilized 8-hydroxyquinoline [21]. These new IMAC ligands allow for greater specificity for certain metal cations, depending on the ligand binding site utilized and strength of the ligand-metal-analyte interaction. Therefore, choosing metal binding amino acids or immobilized ligands specific to the metal cation of interest can increase reproducibility and metal retention by the column.

Intermediate metal ions of copper, nickel, zinc, iron, and gallium are most commonly used for the IMAC isolation of metal binding proteins and phosphopeptides 
[11]. Reliable methods have been developed for the elution of ligands chelated to intermediate metal ions, and the analyte is usually eluted free in solution while the metal ion is retained on the column. However, IMAC has not been used widely for the isolation of naturally occurring metal binding acids that are biosynthetically produced by mycorrhizal fungi in the environment. Moreover, hard metal ions, such as $\mathrm{Fe}^{3+}$ and $\mathrm{Al}^{3+}$, have not been as thoroughly explored using IMAC as other traditionally used intermediate

metals. $\mathrm{Fe}^{3+}$ is not nearly as hard of a metal ion as $\mathrm{Al}^{3+}$ and has recently found common use for the selective enrichment of phosphopeptides [21]. However, because of its especially high charge-size ratio, $\mathrm{Al}^{3+}$ is considered a very hard metal ion and is still rarely used in IMAC.

\subsubsection{Other Applications of IMAC}

IMAC has often been used for phosphopeptide and protein analysis, and the extraction of his-tagged proteins where the histidine residues are inserted recombinantly [11]. Furthermore, metal chelating proteins in wheat and tobacco have been characterized with the use of IMAC [12]. Using in vivo labeled proteins, metal binding proteins in these plants were successfully identified [12]. IMAC has also been utilized in the medical field for cancer detection by isolating and identifying unique proteins in serum that relate to bone metastases [28]. Additionally, IMAC also holds promise to isolate complex mixtures of biological compounds that may be specifically involved in metal ion resistance. Therefore, a wide range of uses exist with the development of an $\mathrm{Al}^{3+}$-IMAC method for naturally occurring ligands. 


\subsubsection{IMAC ligands}

Due to the surge in popularity of IMAC, many new ligands have been developed to increase binding strength and specificity. However, the tridentate ligand iminodiacetic acid (IDA) still remains one of the most commonly used IMAC ligands due to its relative versatility and stability of the metal ion complexes. Recently, development of immobilized nitrilotriacetic acid (NTA), a tetradentate ligand, and also a non-disclosed pentadentate ligand (Affiland, Belgium) have given researchers more options to tailor fit this separation technique to their specific needs [21]. These higher coordination numbers often result in higher metal binding strength with the immobilized ligand and, in most cases, lower protein binding strength, resulting in easier elution of the free protein from the immobilized metal [21]. Both IDA and NTA bind metal cations using a similar mechanism, in which the metal binding to one nitrogen atom and two or three oxygens respectively [21]. As a result, NTA should bind the metal ion more strongly, provided all of the functional groups can acquire the appropriate spatial orientation. However, NTA complexed metal ions may also bind to a protein more weakly than IDA due to fewer coordination sites available on the metal ion [21]. Figure 3.1 shows the structure of IDA and NTA along with some other immobilized ligands that are commercially available. 


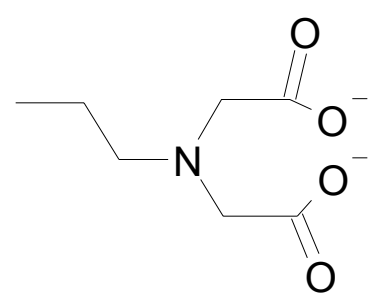

Iminodiacetic Acid (IDA)

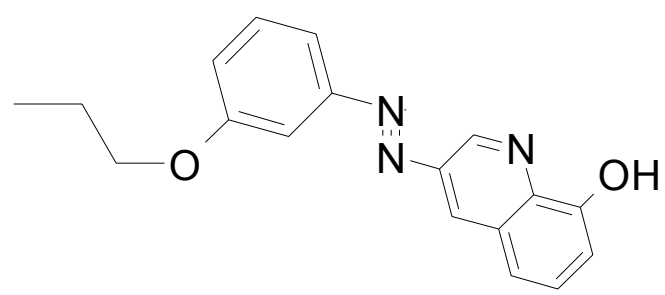

8-Hydroxyquinoline (8-HQ)

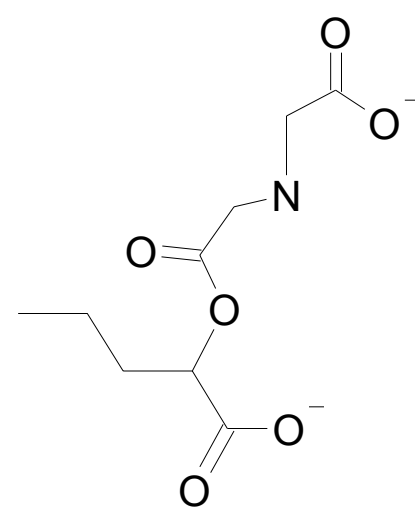

Nitrilotriacetic Acid (NTA)

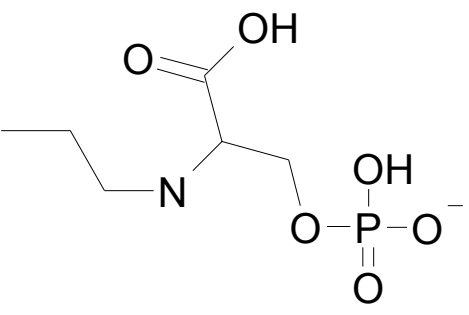

O-Phosphoserine (OPS)

Figure 3.1-Immobilized ligands commonly used in immobilized metal affinity chromatography [21].

Regardless of the exact binding mechanism, the purpose of the IMAC ligand is the same: to immobilize the metal cation strongly enough to avoid bleeding from the column over time as well as leaving the metal cation accessible for analyte binding and elution.

\subsubsection{Hard metal IMAC}

Metals that are classified as hard metals have a high charge density. That is to say they possess a high charge and small radius. Furthermore, hard metals have a low polarizability, meaning their electron cloud is not easily distorted. Metals such as $\mathrm{Fe}^{3+}$ or $\mathrm{Al}^{3+}$ are common examples of hard metals [20]. Because metal ions are electron acceptors, they are frequently referred to as Lewis acids. Therefore, hard metals are also classified as hard acids. In hard/soft acid/base chemistry, it is known that hard acids more readily bind 
to hard bases. The same is true of binding between soft acids and soft bases. Soft metals such as $\mathrm{Cu}^{+}$and $\mathrm{Ag}^{+}$have a low charge and are easily polarized and oxidized and are not suitable for IMAC as they are typically monovalent. Due to the low charge and partial ionic nature of the coordinate covalent bonds, immobilized soft metal ions do not readily bind analyte ligand(s). The most common metals used for IMAC methods have traditionally been the intermediate metals such as $\mathrm{Ni}^{2+}, \mathrm{Zn}^{2+}$, or $\mathrm{Cu}^{2+}$ [21]. The advantage of these intermediate metals is that they are relatively universal in their binding.

Hard acids are often too specific for broad applications. However, hard metal specificity could be advantageous, such as the affinity of $\mathrm{Fe}^{3+}$ for phosphorus containing molecules [11]. Despite the lack of previous data available for hard metals in IMAC, initial experiments have shown that the metal exchange interaction of IMAC with $\mathrm{Al}^{3+}$ can be utilized for the isolation of metal binding exudates [29].

\subsubsection{Rationale for research}

The pigments found in fungal exudates are thought to be important in inferring metal tolerance to vascular plants. Additionally, it has been established that IMAC ligands can bind $\mathrm{Al}^{3+}$ and be used to separate molecules based on their $\mathrm{Al}^{3+}$ affinity [11]. Thus $\mathrm{Al}^{3+}$ IMAC was used for the isolation of $\mathrm{Al}^{3+}$ binding ligands from the fungal exudate mixture. By using IMAC for this selective enrichment of the ligands, components in the exudate mixture that do not bind $\mathrm{Al}^{3+}$ are removed, easing downstream analysis by mass spectrometry. Furthermore, the $\mathrm{Al}^{3+}$ does need to be removed from the pigment ligands in order to examine the ligands' structures. Therefore, $\mathrm{Al}^{3+}$-IMAC can be used for isolation and preparation of Al-binding ligands from biological samples for structural analysis by ESI-MS. 


\subsection{Experimental}

Initially, HiTrap ${ }^{\mathrm{TM}}$ immobilized metal affinity chromatography cartridges, $(1 \mathrm{~mL}$ cartridge volume, Amersham Pharmacia, Piscataway, NJ, USA), which use iminodiacetic acid as the metal immobilizing ligand, were used to isolate metal-binding fungal compounds. IMAC isolations were also performed using nitrilotriacetic acid (NTA) Superflow beads (Qiagen, Alameda, CA, USA) packed in $1 \mathrm{~mL}$ cartridges. These beads

were bought in bulk and packed in house at low pressure in emptied HiTrap ${ }^{\mathrm{TM}}$ cartridges. Constant flow (1 mL/min) and volumes were maintained with a $\mathrm{KD}$ Scientific syringe pump (Holliston, MA, USA). ACS certified aluminum chloride (Fisher Scientific, Pittsburgh, PA, USA) was prepared at a concentration of $10 \mathrm{~m} M$ to load the metal cation onto the IMAC column. A 20\% ethanol (ACS certified, Fisher Scientific, Pittsburgh, PA, USA), $0.1 \%$ acetic acid solution $(\mathrm{pH} \approx 3)(99.99 \%$, Sigma-Aldrich, St. Louis, MO, USA) was used as the IMAC wash solution to overcome non-specific binding of ligands to the bead matrix. The elutors benzenesulfonic acid, 1,3-benzenedisulfonic acid and tiron (4,5dihydroxy-1,3-benzenedisulfonic acid), were from Sigma-Aldrich. Malonic acid was obtained from Sigma-Aldrich (St. Louis, MO, USA) and oxalic acid from J.T. Baker (Phillipsburg, NJ, USA) and imidazole was purchased from Fisher Scientific. Deionized water from an 18.1 Mohm water purification system (Barnstead International, Dubuque, Iowa) was used to prepare all solutions.

\subsubsection{Aluminum binding}

Initially, a qualitative IMAC assay was used to screen for $\mathrm{Al}^{3+}$ binding ligands and assess the viability of this technique. Aluminum chloride was loaded in excess onto the IMAC column to bind all ligand sites. The column was rinsed to remove any unbound $\mathrm{Al}^{3+}$ 
before loading fungal exudates. The column and beads are transparent, allowing retention and elution of the exudates on/from the IMAC column to be detected colorimetrically through the column wall. At this point it was unclear whether this retention was due to non-specific binding or interaction with the immobilized $\mathrm{Al}^{3+}$. Imidazole, the manufacturers suggested elutor, was applied to the column but did not elute the pigments. To minimize the retention through non-specific hydrophobic interactions with the bead matrix, varying concentrations of organic solvents (acetonitrile and ethanol) were passed through the IMAC column but were unsuccessful in eluting the exudate. Many concentrations of commonly used elutors and known metal binding compounds, such as small organic acids and sulfonic acids, along with increasing the $\mathrm{pH}$ of these potential elutors to 11, were used before a successful and reproducible elution was accomplished. These elutors included, EDTA, imidazole, citrate, eriochrome cyanine, 8hydroxyquinoline, malonic acid, oxalic acid, benzenesulfonic acid, 1,3-benzenedisulfonic acid, and 4,5-dihydroxy-1, 3-benzenedisulfonic acid (tiron), $\mathrm{NaOH}, \mathrm{NH}_{4} \mathrm{Cl}$, and $\mathrm{NH}_{4} \mathrm{OH}$. Once appropriate solutions were found for the extraction and elution of the pigments, the next step was to quantitatively evaluate the effectiveness of the different elutors.

The first step in evaluating the IMAC protocol was to quantitate the $\mathrm{Al}^{3+}$ binding of the IDA and NTA IMAC beads. The binding capacity was determined by loading the column with $\mathrm{Al}^{3+}$, washing away unbound $\mathrm{Al}^{3+}$, removing the $\mathrm{Al}^{3+}$, and measuring the $\mathrm{Al}^{3+}$ concentration with ICP-AES. Three control trials were performed using the conditions: no $\mathrm{Al}^{3+} /$ no exudate for background, no $\mathrm{Al}^{3+} /$ exudate loaded for $\mathrm{Al}^{3+}$ contribution from exudate, and $\mathrm{Al}^{3+}$ loaded/no exudate for $\mathrm{Al}^{3+}$ binding and elution without ligand present. 
Furthermore, both $\mathrm{Al}^{3+}$ and exudate were loaded on the IMAC column to determine $\mathrm{Al}^{3+}$ binding under experimental conditions (Table 3.1).

\begin{tabular}{ccccccc}
\hline Purpose & Wash & $\begin{array}{c}\text { Load } \\
\mathbf{A l}^{3+}\end{array}$ & Wash & $\begin{array}{c}\text { Load } \\
\text { Exudate }\end{array}$ & Wash & $\begin{array}{c}\text { Elute } \\
\text { Exudates }\end{array}$ \\
\hline Background Control & + & - & - & - & - & + \\
Non-Specific Binding Control & + & - & - & + & + & + \\
Background Control w/Al ${ }^{3+}$ & + & + & + & - & - & + \\
Experimental & + & + & + & + & + & + \\
\hline
\end{tabular}

Table 3.1-Experimental conditions for the evaluation of $\mathrm{Al}^{3+}$ binding to the IMAC column. Trials performed with (+) and without (-) $\mathrm{AlCl}_{3}$ and exudate as needed. Concentrations and volumes were: Wash $=4 \mathrm{~mL}, 20 \%$ ethanol, $0.1 \%$ acetic acid; $\mathrm{AlCl}_{3}=4 \mathrm{~mL}, 10 \mathrm{mM}$; exudate $=1 \mathrm{~mL}$; elution= $M \mathrm{NH}_{4} \mathrm{OH}$.

The addition of $\mathrm{Al}^{3+}$-free fungal exudates determined if any $\mathrm{Al}^{3+}$ contamination existed in the unseparated exudates and if it hindered the ability to elute $\mathrm{Al}^{3+}$ from the IMAC column. In the no $\mathrm{Al}^{3+} /$ no exudate trial, the IMAC column was washed with $4 \mathrm{~mL}$ wash solution (20\% ethanol, $0.1 \%$ acetic acid) and $4 \mathrm{~mL}$ elution solution $\left(1 \mathrm{M} \mathrm{NH}_{4} \mathrm{OH}\right)$ and the $1 \mathrm{~mL}$ fraction collected for the. The procedure for $\mathrm{Al}^{3+} /$ exudate trials involved washing the column with $4 \mathrm{~mL}$ wash solution, charging the column with $4 \mathrm{~mL}$ of $10 \mathrm{mM} \mathrm{AlCl}, 4 \mathrm{~mL}$ wash solution, $1 \mathrm{~mL}$ exudate, $4 \mathrm{~mL}$ wash solution, $4 \mathrm{~mL}$ elution solution $\left(1 \mathrm{M} \mathrm{NH}_{4} \mathrm{OH}\right)$ and the $1 \mathrm{~mL}$ fraction collected. The control trials were performed using this same process except with the omission of the $\mathrm{Al}^{3+}$ and/or exudate loadings as appropriate. These column loading conditions are summarized in Table 3.1. All separations were performed in triplicate to establish the reproducibility of elution. The IMAC columns were eluted with 1 $M \mathrm{NH}_{4} \mathrm{OH}$ and fractions collected in each trial. During all IMAC steps, a syringe pump set at a flow rate of $1.0 \mathrm{~mL} / \mathrm{min}$ was used to main constant flow through the column. The eluent fractions were analyzed for $\mathrm{Al}^{3+}$ on the ICP-AES to determine the binding capacity of $\mathrm{Al}^{3+}$ on the IMAC column and how it may vary in the presence of exudate. A standard curve was prepared for ICP-AES analysis using solutions of $0.1,1,10$, and $100 \mathrm{ppm} \mathrm{Al}^{3+}$ 
and $\mathrm{Cu}^{2+}$ in $20 \%$ ethanol and $0.1 \%(\mathrm{v} / \mathrm{v})$ acetic acid. The addition of ethanol was used to match the IMAC wash solution composition. $10 \mathrm{ppm}$ copper was used as an internal standard in all samples to account for any matrix or instrumental variance. ICP-AES standards were prepared using atomic absorption grade aluminum and copper standards from Fisher Scientific (Pittsburgh, PA, USA). For the ICP, the sample flow rate was 10 $\mathrm{mL} / \mathrm{min}$, the power was 1300 watts, and the 309.2 and $223.0 \mathrm{~nm}$ lines were used for aluminum and copper detection respectively.

\subsubsection{Exudate binding}

Once it was determined that IMAC was effective for immobilizing $\mathrm{Al}^{3+}$ and isolating the exudate (Figure 3.2, Table 3.3), it was necessary to determine how much of the bound exudate could be removed from the IMAC column. The IMAC column was loaded with $4 \mathrm{~mL}$ of $10 \mathrm{mM} \mathrm{AlCl}$, $4 \mathrm{~mL}$ of wash solution, $1 \mathrm{~mL}$ of fungal exudate, then 7 $\mathrm{mL}$ of wash solution. During this wash step, the void volume was discarded and seven 1 $\mathrm{mL}$ fractions collected. Then, the column was washed with $4 \mathrm{~mL}$ of $1 \mathrm{MNH}_{4} \mathrm{OH}$ and four $1 \mathrm{~mL}$ fractions collected. For the control, this loading, washing, and eluting process was repeated, but the column was not charged with $\mathrm{Al}^{3+}$. Each of these trials had an accompanying blank trial performed where liquid growth media was used rather than exudate, serving as UV-Vis blanks for the respective fraction.

As a check for fungal ligand specificity for the $\mathrm{Al}^{3+}$-IMAC system, IMAC separations were performed using $10 \mathrm{~m} M$ 8-hydroxyquinoline (8-HQ) (Fisher Scientific, Pittsburgh, PA, USA) and $10 \mathrm{~m} M$ eriochrome cyanine RC (EC) (practical grade) from Sigma as analyte ligands. Using 8-HQ and EC as model compounds and fungal exudates as an unknown, extractions were performed in parallel on IMAC columns with and without 
$\mathrm{Al}^{3+}$. One IMAC column was loaded with $4 \mathrm{~mL} 10 \mathrm{mM} \mathrm{AlCl}$, the other column with no metal. Both columns were then flushed with $4 \mathrm{~mL}$ wash solution, loaded with $1 \mathrm{~mL}$ of the respective ligand/fungal exudates and washed with $4 \mathrm{~mL}$ of wash solution. Elutors were then applied to the IMAC columns: The first $1 \mathrm{~mL}$ (void volume) was discarded and subsequent $1 \mathrm{~mL}$ fractions collected. All collected fractions were then analyzed on a single beam UV-Vis spectrophotometer (Hewlett Packard 8453, Wilmington, DE, USA). Since

all of these $\mathrm{Al}^{3+}$-binding ligands absorb in the UV-visible range, the absorbance intensity provided a relative comparison of how much ligand was retained in the presence and absence of $\mathrm{Al}^{3+}$.

\subsubsection{Elution}

Initial probing for ideal elution conditions was performed using varying concentrations of imidazole and EDTA. Some of these elutors removed small amounts of the fungal exudates, but substantial amounts of exudates remained on the $\mathrm{Al}^{3+}$-IMAC column. A second series of elutions with carboxylic and sulfonic acids was performed. These included $20 \mathrm{~m} M$ solutions of malonic acid, oxalic acid, benzenesulfonic acid, 1,3benzenedisulfonic acid, and 4,5-dihydroxy-1, 3-benzenedisulfonic acid (tiron). These elutors also proved ineffective under native conditions (dissolved in $\mathrm{dI}_{\mathrm{H}} \mathrm{O}$ ), but were more effective in basic solutions ( $\mathrm{pH} 9-11)$. To compare the efficiency of the elutors, a series of extractions were performed in a systematic manner using elutors at $\mathrm{pH} 11$ with $\mathrm{NaOH}$ and $\mathrm{NH}_{4} \mathrm{OH}$ used to adjust the $\mathrm{pH}$ separately. IMAC columns were again charged with $4 \mathrm{~mL}$ of $10 \mathrm{mM} \mathrm{AlCl}$, washed with $4 \mathrm{~mL}$ wash solution, loaded with $1 \mathrm{~mL}$ exudate, washed with $4 \mathrm{~mL}$ of wash solution, and $4 \mathrm{~mL}$ of the selected elutor was applied to the column. The void volume was allowed to pass through the cartridge before collecting a 1 
$\mathrm{mL}$ volume fraction of the eluted pigment front. The following elutors were used at a concentration of $20 \mathrm{mM}$ and adjusted to $\mathrm{pH} 11$ with $\mathrm{NaOH}$ and $\mathrm{NH}_{4} \mathrm{OH}$ separately: oxalic acid, malonic acid, 1,3-benzenedisulfonic acid, benzenesulfonic acid, imidazole, and tiron. $\mathrm{NaOH}, \mathrm{NH}_{4} \mathrm{Cl}$ and $\mathrm{NH}_{4} \mathrm{OH}$ solutions were $1 M$ in solution and $\mathrm{NH}_{4} \mathrm{Cl}$ was left at its native $\mathrm{pH}$ without adjustment. A blank was prepared for each aliquot by running an extraction in parallel under the same conditions except liquid growth media was loaded in place of the exudate and fraction collected. A standard exudate sample was provided by loading the fungal exudates onto an $\mathrm{Al}^{3+}$-IMAC column and eluting with $1 M \mathrm{NH}_{4} \mathrm{OH}$. This prepared standard corresponds to the IMAC purified exudate of interest for a more accurate representation than the untreated exudate mixture. The different elutors were added to separate aliquots of this exudate standard to make elutor-exudate standards.

The absorbance values of the IMAC fractions were normalized to minimize the environmental effects caused by changes of the solution composition and interactions between the elutor and fungal exudates. In most cases, summing together the intensity of the elutor spectra and exudates spectral peaks did not produce the elutor-exudates standard spectra. This non-additive behavior is caused by binding, masking, and other interactions of the exudate and the elutor. When the exudate standard and elutor spectra were overlaid, a crossover point typically appeared at a longer wavelength $(400-450 \mathrm{~nm})$. At this point, the intensity of the elutor spectrum and the exudates standard spectrum were the same and the slopes had the same sign. The intensity of the sample spectrum $\left(\mathrm{A}_{\mathrm{s}}\right)$ was divided by the intensity of the exudates standard $\left(\mathrm{A}_{\mathrm{e}}\right)$ at this crossover point wavelength to normalize for the elutor absorbance and the elutor-exudates interactions. All of these values were divided by the value obtained for ammonium hydroxide $\left(\mathrm{A}_{\mathrm{ah}}\right)$, which had the greatest recovery, and 
is shown below. By performing this correction, a final, normalized value $\left(\mathrm{N}_{\mathrm{v}}\right)$ was obtained that accounts for environmental effects and elutor-exudate interactions.

$$
\mathrm{N}_{\mathrm{v}}=\left(\mathrm{A}_{\mathrm{s}} / \mathrm{A}_{\mathrm{e}}\right) / \mathrm{A}_{\mathrm{ah}}
$$

In order to observe the magnitude of change in elutor effectiveness as a function of $\mathrm{pH}$, a number of IMAC separations were performed using $20 \mathrm{~m} M$ tiron as the elutor over a $\mathrm{pH}$ range of 4-11. IMAC columns were loaded with $10 \mathrm{mM} \mathrm{AlCl}$, washed with $4 \mathrm{~mL}$ wash solution, loaded with $1 \mathrm{~mL}$ of exudate, and washed again with $4 \mathrm{~mL}$ wash solution. Finally, $4 \mathrm{~mL}$ of tiron at $\mathrm{pH} 4$ was added. The void volume $(1 \mathrm{~mL})$ was allowed to pass though the column before collecting $1 \mathrm{~mL}$ eluted fractions. The blank, which was run sideby-side with the sample column, was prepared identically as the sample, but with blank fungal media rather then fungal exudates added to this column. This procedure was repeated using $20 \mathrm{~m} M$ tiron adjusted to $\mathrm{pH} 5,6,7,8,9,10$, and 11 with $\mathrm{NH}_{4} \mathrm{OH}$. All collected fractions were analyzed with the UV-Vis spectrophotometer, using each fraction's respective blank to zero the instrument.

$\mathrm{UV}-\mathrm{V}$ is absorbance measurements can be used to determine concentration, but are sensitive to environmental changes. Altering the $\mathrm{pH}$, solvent content, etc. of a solution can lead to shifts in both absorbance wavelength and intensity of the absorbing species. These shifts can be contributed to masking effects by the altered matrix or chemical property changes that may occur to the compound of interest. Therefore, when quantitating the pigment concentration in different solvents and buffer solutions, the change in absorbance due to environmental changes must be accounted for. 
A series of elutor and non-IMAC isolated fungal exudate mixtures were made and analyzed using a UV-Vis spectrophotometer to assess potential optical interactions. Exudate stock was diluted 200:1 to stay within the reasonable intensity range. Aliquots of this exudate were then combined 1:1 (v/v) with each elutor. Liquid media in water was used as the blank for each analysis. The samples consisted of each elutor by itself, exudate by itself, and each elutor combined with exudate. These spectra were then compared to determine if the elutor alters the absorbance wavelength and intensity of the pigment.

\subsubsection{Non-specific Binding}

Ideally, solid-phase extractions and chromatographic separations should separate based on a single mechanism. In chromatography, if factors other than the desired mode of separation are present, band broadening is usually observed and is most pronounced with gradient elution. In solid phase extraction, non-specific binding reduces the purity of the isolated compound(s). The cross-linked sepharose beads and linking group for the IMAC ligand provide a mildly hydrophobic surface for the exudates to interact with, thus causing additional retention based on the compounds hydrophobicity. In order to overcome this potential problem, the elutor was prepared with a range of ethanol concentrations to determine the degree of hydrophobic interaction between the fungal exudates and stationary phase matrix. Ethanol was chosen because it was the organic component in the manufacturers recommended storage solution. The concentration of ethanol in each solution was varied between $0-50 \%$ in $10 \%$ steps with the concentration of elutor being held constant. Separate IMAC extractions were performed using the increasing concentrations of ethanol solutions as elutors with the IMAC column being 
stripped between each trial. The fractions from each elution were collected and analyzed on the UV-Vis to determine if the exudate present in each fraction varied based on ethanol concentration. Blanks for UV-Vis analysis were prepared as before, by running a second IMAC column in parallel and loading liquid media rather than exudates.

\subsection{Results and Discussion}

\subsubsection{Aluminum binding}

It was necessary to determine the $\mathrm{Al}^{3+}$ binding capacity of the IDA and NTA stationary phases used because the $\mathrm{Al}^{3+}$ must be retained in reasonable quantities for the extraction of $\mathrm{Al}^{3+}$ binding ligands. Very low binding capacities would provide few sites for retention of $\mathrm{Al}^{3+}$, causing decreased retention of $\mathrm{Al}^{3+}$ binding compounds from the analyte. Without the retention of $\mathrm{Al}^{3+}$, the IMAC stationary phase can behave as an ion exchange resin and result in a relative increase in the binding of non-specific compounds. Because $\mathrm{Al}^{3+}$ IMAC has received little development, literature values for the $\mathrm{Al}^{3+}$ binding capacities of the IDA and NTA stationary phases were not available. The binding capacity of $\mathrm{Al}^{3+}$ was measured to be about $12 \mu \mathrm{mol} \mathrm{Al}^{3+} / \mathrm{mL}$ matrix $(\sigma=0.45)$ using ICP-AES, which is reasonably agreeable with the manufacturer values for copper (II) binding $(23 \mu \mathrm{mol}$ $\mathrm{Cu}^{2+} / \mathrm{mL}$ matrix from Amersham Biosciences, Piscataway, NJ, USA). This suggests that not all binding sites on the IMAC column are saturated due to the lower binding capacity for $\mathrm{Al}^{3+}$ versus $\mathrm{Cu}^{2+}$. It is expected that the IDA matrix will bind less $\mathrm{Al}^{3+}$ than $\mathrm{Cu}^{2+}$ because of the difference in the nature of $\mathrm{Al}^{3+}$ and $\mathrm{Cu}^{2+}$. Due to the intermediate and soft bases that make up the binding site of the IDA, hard metals such as $\mathrm{Fe}^{3+}$ and $\mathrm{Al}^{3+}$ are retained in smaller quantities and are bound less strongly than softer metals [21]. Secondly, $\mathrm{Al}^{3+}$ has slower binding kinetics than the other metal ions used for IMAC $[21,26]$. Finally, 
topological heterogeneity of the bead matrix and diversity in the microenvironments contribute to the behavior among different metal cations on the IMAC column [11]. Differing distances between ligands arms and their relative orientation will change the binding capacity of an IMAC column for different metals [11]. Both the IDA and NTA IMAC columns that were charged with $\mathrm{Al}^{3+}$ showed an effectively equal loading capacity. ICP-AES analysis of fractions from $\mathrm{Al}^{3+}$ free columns showed negligible $\mathrm{Al}^{3+}$ contribution

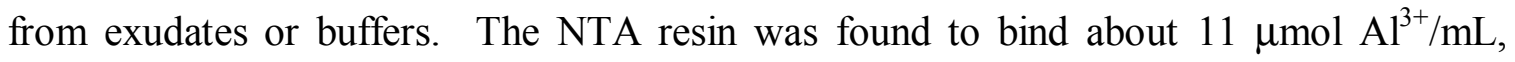
indicating the amount of $\mathrm{Al}^{3+}$ binding is similar for both IMAC resins. Not only do both IMAC ligands show a comparable binding capacity for $\mathrm{Al}^{3+}$, but the exudates elute from both columns under similar conditions. This should not be surprising since both ligands utilize acetic acid groups as their binding site. Even though NTA has an additional acetate site per ligand arm; experimental results show a similar degree of $\mathrm{Al}^{3+}$ retention by NTA as with IDA. $\mathrm{Al}^{3+}$ is a small, highly charged cation and does not exhibit stronger binding with the additional acetate group. A plausible explanation for the equivalent binding with IDA and NTA is that the $\mathrm{Al}^{3+}$ is not accessible to the additional acetate to provide an appropriate spatial orientation for alignment with an empty $\mathrm{Al}^{3+}$ orbital. In other words, the small size of $\mathrm{Al}^{3+}$ and structure of NTA (Figure 3.1) is too sterically hindered for tetradentate coordination. This is demonstrated in Table 3.2, which contains the stability constants for NTA with various metals in solution.

\begin{tabular}{cc}
\hline Metal & Stability Constant $(\log \mathbf{K})$ \\
\hline $\mathrm{Al}^{3+}$ & 10 \\
$\mathrm{Cu}^{2+}$ & 12.68 \\
$\mathrm{Ni}^{2+}$ & 11.26 \\
\hline
\end{tabular}

Table 3.2-Stability constants for nitrilotriacetic acid with various metal cations [32]. 


\subsubsection{Exudate binding}

Approximately $90 \%$ of the fungal exudates were retained on the $\mathrm{Al}^{3+}$-IMAC column. As shown in Figure 3.2, only a small portion of the pigment was not retained on the $\mathrm{Al}^{3+}$ activated column, and most of the pigment eluted after addition of the elutor solution. On the other hand, most of the pigment was not retained on the $\mathrm{Al}^{3+}$ free IMAC column and eluted in the first 2 fractions, while a small amount was released from the column by the elutor solution. Therefore, the data indicates that binding is through $\mathrm{Al}^{3+}$ and most of the pigments in the exudate mixture are shown to be chelators of $\mathrm{Al}^{3+}$. It should be noted that this behavior was observed to be reproducible using colorimetric detection. However, UV-Vis analysis data was not extensive enough to obtain the standard deviation.

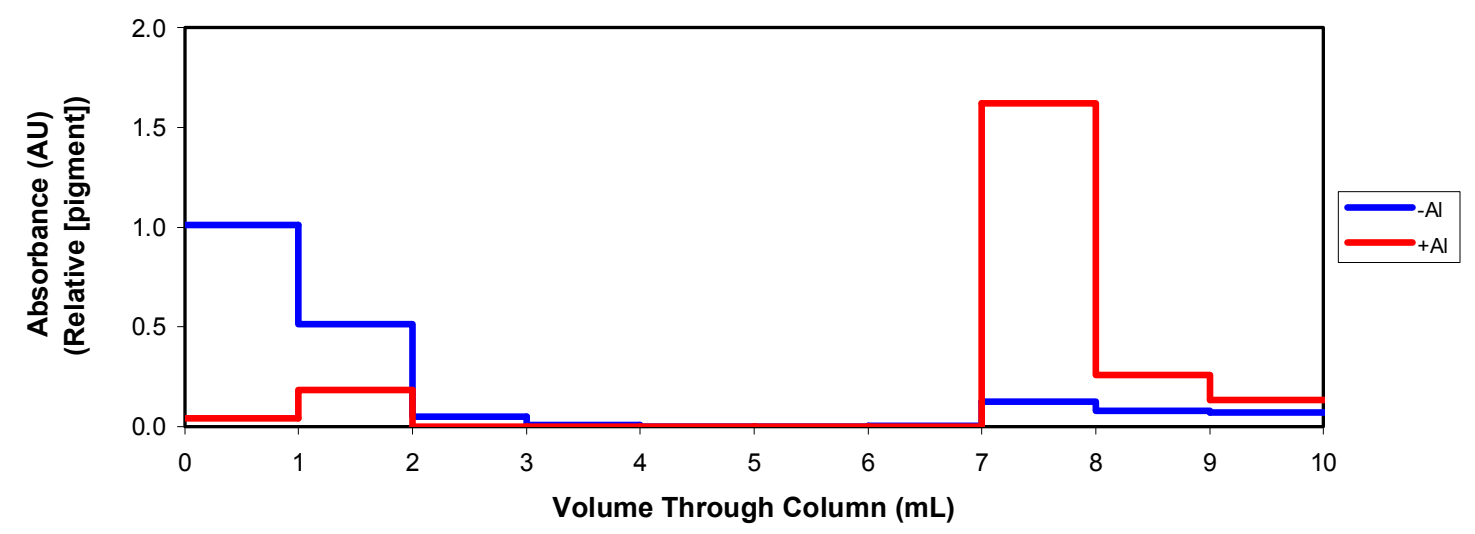

Figure 3.2-UV-Vis data from fractions collected sequentially from IMAC elutions. -Al represents IMAC separation performed with no $\mathrm{Al}^{3+}$ loaded on the column. + $\mathrm{Al}$ represents IMAC separation performed with $4 \mathrm{~mL} 10 \mathrm{mM} \mathrm{AlCl} 3$ loaded onto column. Fraction volumes 0-7 were flushed with $20 \%$ ethanol, $7-10$ were eluted with $1 M \mathrm{NH}_{4} \mathrm{OH}$.

$\mathrm{Al}^{3+}$ charged columns had a higher capacity for fungal exudates than the known $\mathrm{Al}^{3+}$ ligands $\mathrm{EC}$ and 8-HQ, which is illustrated by the larger difference in intensity between 
the $\mathrm{Al}^{3+}$ free and $\mathrm{Al}^{3+}$ charged trials for exudate as opposed to those for the other ligands (Table 3.3). The magnitude of difference between the ligand collected from an IMAC column charged with $\mathrm{Al}^{3+}$ and an $\mathrm{Al}^{3+}$ free column was much greater for the fungal exudate than either $\mathrm{EC}$ or 8-HQ, which are known to bind $\mathrm{Al}^{3+}[20]$. Therefore, the fungal exudates have a high affinity for $\mathrm{Al}^{3+}$ compared with the model ligands, EC and 8-HQ, especially at low $\mathrm{pH}$ and may act in a similar manner to other known ligands to provide protection from $\mathrm{Al}^{3+}$ toxicity $[2,20]$.

\begin{tabular}{lc}
\hline & Retained ligand \\
\cline { 2 - 2 } \multicolumn{1}{c}{ Ligand } & Unretained ligand \\
\hline 8-Hydroxyquinoline & 1.2 \\
Eriochrome cyanine & 3.9 \\
Fungal exudate & 5.6 \\
\hline
\end{tabular}

Table 3.3-Absorbance data shows ratio of ligand retained on $\mathrm{Al}^{3+}$ loaded column versus ligand that passes through column unretained. All conditions were the same for each trial except for type of ligand loaded.

\subsubsection{Elution}

The strength of interaction between fungal exudates and $\mathrm{Al}^{3+}$ was so strong that all initial attempts to elute the complex from the column at low $\mathrm{pH}$ failed. An initial concern was that during elution at higher $\mathrm{pH}$, precipitation of polymeric $\mathrm{Al}_{\mathrm{x}}(\mathrm{OH})_{\mathrm{y}}$ species could be problematic, but such precipitates were not observed due to aluminums amphoteric properties [20]. Once it was concluded that the pigment could not be eluted at low $\mathrm{pH}$, the elution efficiency of a number of elutors were tested over a $\mathrm{pH}$ range of 4-11. Tiron proved to be an effective elutor, and the $\mathrm{pH}$ dependence of its elution efficiency is shown in Figure 3.3. Elution was very inefficient over the $\mathrm{pH}$ range 4-8, possibly due to solubility issues, 
but recovery did increase greatly between $\mathrm{pH} 9$ and 11 due to decreased ability of $\mathrm{Al}^{3+}$ to complex the immobilized or analyte ligands at higher $\mathrm{pH}$.

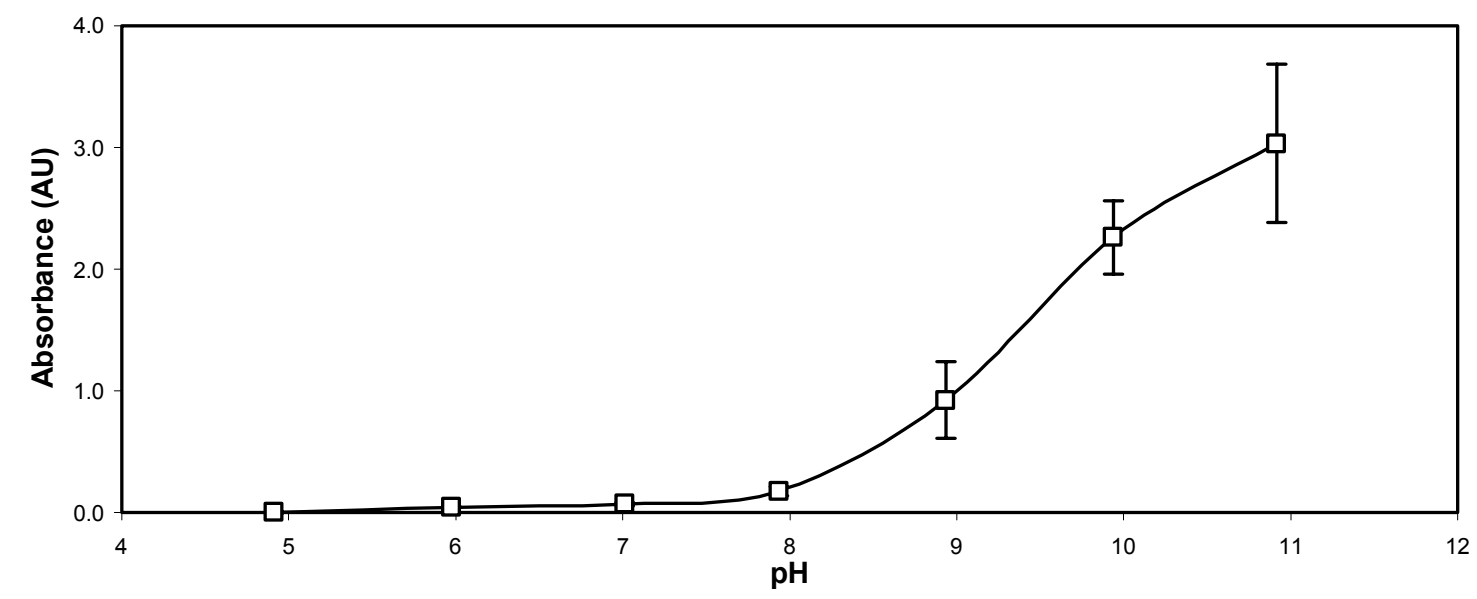

Figure 3.3-Tiron $\mathrm{pH}$ adjusted with $\mathrm{NH}_{4} \mathrm{OH}$. IMAC column loaded with $4 \mathrm{~mL} 10 \mathrm{mM}$ $\mathrm{AlCl}_{3}$ and $1 \mathrm{~mL}$ exudate. Eluted performed at $1 \mathrm{~mL} / \mathrm{min}$. with $20 \mathrm{~m} M$ tiron solution at respective $\mathrm{pH}$. Column was reloaded between trials. Figure points are average of five trials.

Because $\mathrm{Al}^{3+}$ is a hard metal ion, this observation is consistent with previous reports that elution with hard metal ions is most effective at high pHs [11]. When $\mathrm{NH}_{4} \mathrm{OH}$ was used to adjust the $\mathrm{pH}$ of the elutor, the elution efficiency was increased (Table 3.4).

\begin{tabular}{lcc}
\hline \multicolumn{1}{c}{ Elutor } & \multicolumn{2}{c}{ Elution efficiency } \\
\hline & $\mathbf{N a O H}$ & $\mathbf{N H}_{\mathbf{4}} \mathbf{O H}$ \\
\hline Oxalic Acid & 0.60 & 0.64 \\
Malonic Acid & 0.60 & 0.57 \\
Imidazole & 0.22 & 0.38 \\
Benzenesulfonic Acid & 0.12 & 0.33 \\
1,3-Benzenedisulfonic Acid & 0.00 & 0.00 \\
Tiron & 0.24 & 0.58 \\
$\mathrm{NH}_{4} \mathrm{OH}$ & ----- & 1.00 \\
$\mathrm{NH}_{4} \mathrm{Cl}$ & --- & 0.00 \\
$\mathrm{NaOH}^{-}$ & 0.34 & ---- \\
\hline
\end{tabular}

Table 3.4-The amount of pigment eluted from the IMAC cartridge was measured for different elutors. The $\mathrm{pH}$ of the elutor solutions was adjusted with $\mathrm{NaOH}$ or $\mathrm{NH}_{4} \mathrm{OH}$. The concentration of the elutors was $20 \mathrm{~m} M$, while the concentration of the $\mathrm{NH}_{4} \mathrm{OH}, \mathrm{NH}_{4} \mathrm{Cl}$, and $\mathrm{NaOH}$ was $1 M$ when they were used individually in solution. 
$\mathrm{NaOH}, \mathrm{NH}_{4} \mathrm{Cl}$, and $\mathrm{NH}_{4} \mathrm{OH}$ were investigated to determine whether the elution mechanism was simply a factor of $\mathrm{pH}$ or due to the presence of specific ions. $\mathrm{NH}_{4} \mathrm{OH}$ by itself proved to be the most effective elutor and was more efficient than $\mathrm{NaOH}$ and $\mathrm{NH}_{4} \mathrm{Cl}$, suggesting that both the ammonia and hydroxide ion are required for elution. The superior elution efficiency of $\mathrm{NH}_{4} \mathrm{OH}$ and general behavior of the other elutors was reproducible throughout the course of this work. The data in Table 3.4 reflects one trial of the elutor panel performed against a single set of prepared elutor and exudates standards. Because both $\mathrm{NH}_{3}$ and $\mathrm{OH}^{-}$are hard bases, it is logical that they should both play a role in the effective elution of $\mathrm{Al}^{3+}[20]$. For our application, $\mathrm{NH}_{4} \mathrm{OH}$ is the elutor of choice because it is effective at removing exudates, is volatile, and does not interfere with absorbance measurements.

Data obtained from NTA trials also exhibit the same dependence of elution on $\mathrm{pH}$. This elution mechanism resembles that mentioned by Jerker Porath [11] with regards to the ions introduced during a $\mathrm{pH}$ adjustment being responsible for displacing the metal. In this mechanism of $\mathrm{pH}$ adjustment, as the concentration of $\mathrm{H}^{+}$is increased, the analyte ligand is displaced from the immobilized metal by the $\mathrm{H}^{+}$due to the protonation of the analyte ligand. Here, however, the $\mathrm{pH}$ is increased, leading to an increased concentration of $\mathrm{OH}^{-}$, which bind to the $\mathrm{Al}^{3+}$ while the negatively charged immobilized chelator is left behind. Furthermore, deprotonated and uncomplexed exudates continue off the IMAC column. The removal of $\mathrm{Al}^{3+}$ from the column was confirmed by loading the column with an eriochrome cyanine (EC) solution. EC is a colorimetric indicator that is red in solution when unbound but turns deep purple when complexed to a metal. When EC was loaded it did not change colors, indicating $\mathrm{Al}^{3+}$ was absent. As a control, it was confirmed that the 
EC indicator did change color when loaded on the $\mathrm{Al}^{3+}$ charged column. Additionally, the presence of the fungal exudates did not affect the removal of $\mathrm{Al}^{3+}$ from the column, as the same amount of $\mathrm{Al}^{3+}$ was recovered whether the fungal exudates were present or not.

Accounting for environmental effects during UV-Vis quantitation is an important consideration. UV-Vis absorbance is based on a sample's capacity to absorb radiation of a given wavelength. If the structural character of the sample is altered by the addition of a new compound, the samples observed absorbance is likely to change as well. This factor must be addressed when using absorbance for quantitation. According to Beer's law, the concentration is inversely related to the molar absorptivity which is a property inherent to each compound and relates to how strongly a compound absorbs radiation. Therefore, should the compound or its properties change, so will it's observed concentration at a given wavelength.

Preparing mock samples to determine potential environmental effects provided insight into what bias elutors may have on absorbance readings. Because the batch to batch variability has not yet been evaluated and the chromophores present are unknown, a strict standard could not be formed for universal comparison. This technique, however, was sufficient to provide a relative comparison of elutors' effect on exudates absorbance.

The exudates showed some level of absorbance shift when combined with each elutor except for $\mathrm{NH}_{4} \mathrm{OH}$. Malonic and oxalic acid, imidazole, and ammonium hydroxide showed no absorbance by themselves above $\lambda=250 \mathrm{~nm}$. Benzenesulfonic acid, 1,3benzensulfonic acid, and tiron each absorb in the $>250 \mathrm{~nm}$ range. Tiron especially has intense absorbance in the lower wavelength region $(<\lambda=400 \mathrm{~nm})$. Figure 3.4A-C contain representative spectra for the mixtures analyzed. Ammonium hydroxide's lack of spectral 
interference is another benefit to its application as an IMAC elutor for $\mathrm{Al}^{3+}$. During the course of $\mathrm{UV}-\mathrm{V}$ is analysis of fungal exudate, it is clear that the exudate is very prone to environmental effects. Therefore, utilizing an elutor that minimizes these effects is advantageous. Figure 3.5 demonstrates the correlation between standard mixtures and an IMAC isolated fraction. The overlap shows that during the course of the IMAC process, the resulting eluent can still be compared with the unseparated exudate. The small change in intensity can be attributed to the inability to match the exact dilution factor of the controls with that of the eluent.

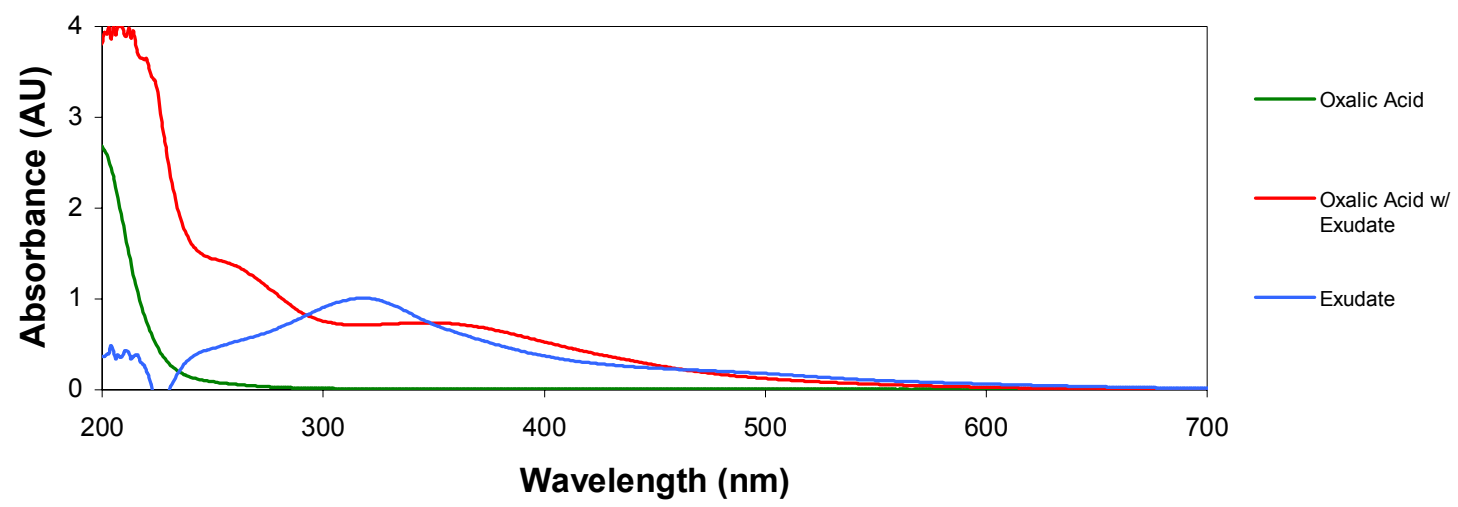

Figure 3.4A-Absorbance spectra of different solutions to compare exudate with and without the presence of $20 \mathrm{~m} M$ oxalic acid.

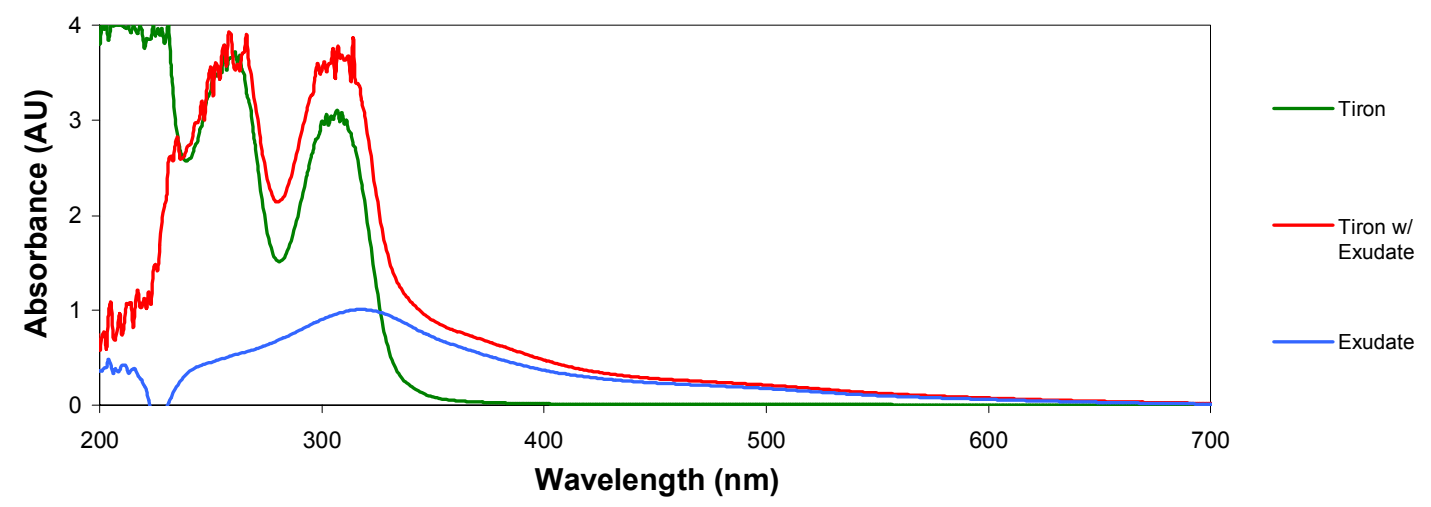

Figure 3.4B-Absorbance spectra of different solutions to compare exudate with and without the presence of $20 \mathrm{~m} M$ tiron. 


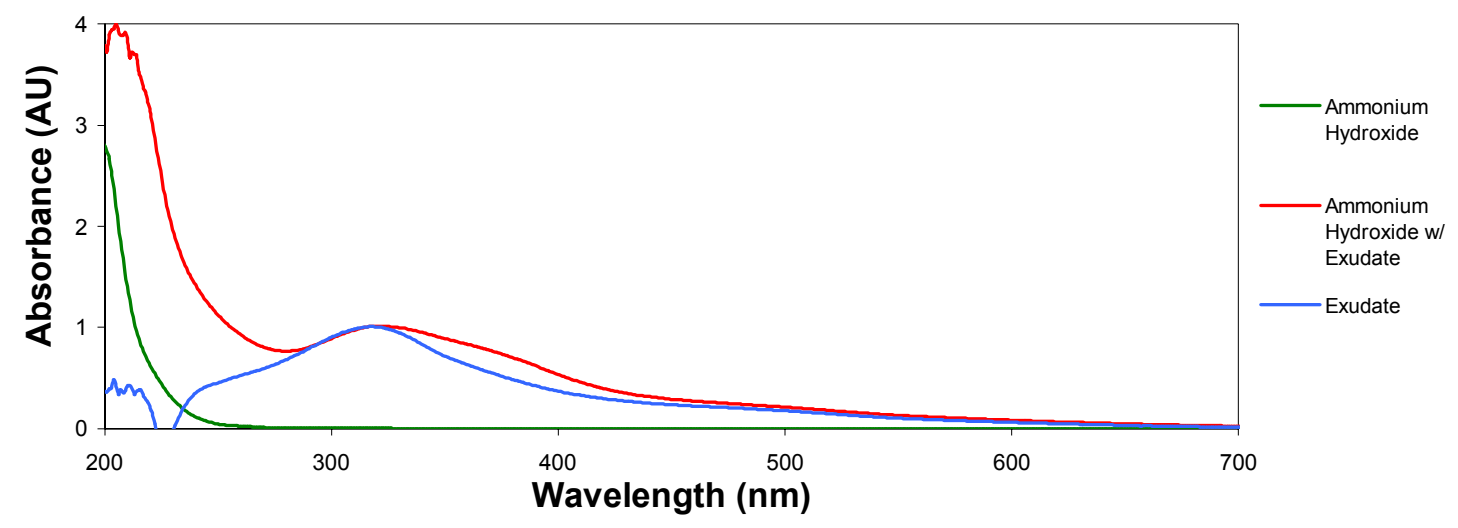

Figure 3.4C-Absorbance spectra of different solutions to compare exudate with and without the presence of $1 \mathrm{MNH}_{4} \mathrm{OH}$.

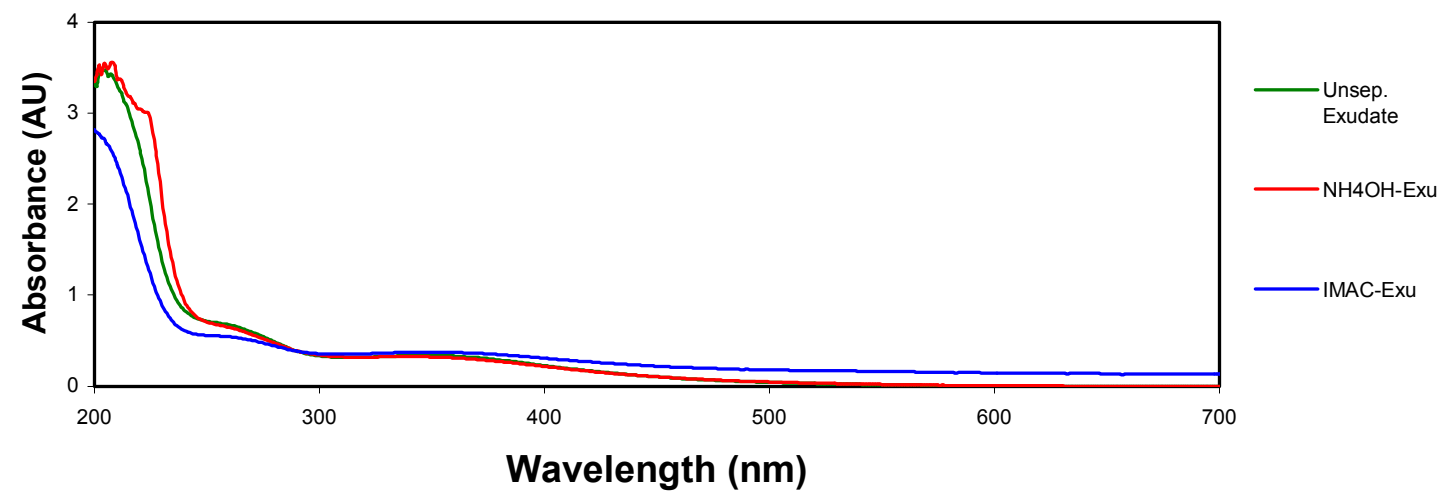

Figure 3.5-Absorbance comparison of IMAC fractions and control mixtures to determine change in absorbance during IMAC isolation.

\subsubsection{Non-Specific Binding}

To insure that the retention of the fungal exudates on the $\mathrm{Al}^{3+}$-IMAC column was due to interactions with the $\mathrm{Al}^{3+}$ and not caused by non-specific interactions, the analyte binding selectivity with $\mathrm{Al}^{3+}$ was verified with a control in which the experimental parameters were identical to the $\mathrm{Al}^{3+}$-IMAC separations except the IMAC column was not charged with $\mathrm{Al}^{3+}$.

It appears that there is trace non-specific binding that occurs during IMAC isolation. When the elutor solution includes ethanol, the eluted exudate is removed from the column in a much more focused band than that eluted by an aqueous solution. Column 
elution using aqueous solutions resulted in greater diffusion and, therefore, band broadening due to these hydrophobic interactions. Consequently, including organic solvents in the elutor solution allows for more efficient elution using a smaller volume of elutor solution. However, the difference in elutor efficiency between the different organic concentrations was negligible. This result shows that the hydrophobic interactions are minor and can be overcome with a relatively low concentration of ethanol. Elutor solutions containing $20 \%$ ethanol were chosen since they give sufficient organic composition to overcome the weak hydrophobic interactions but will not hinder further analysis of the IMAC fraction.

\subsection{Conclusions}

\subsubsection{IMAC suitability for aluminum}

Through the course of this study, successful isolation, elution, and collection of $\mathrm{Al}^{3+}$-binding fungal exudates was accomplished. This work demonstrates that NTA and IDA effectively and similarly bind $\mathrm{Al}^{3+}$. Although the binding capacity of $\mathrm{Al}^{3+}$ on these ligands is lower than for intermediate metals, the $\mathrm{Al}^{3+}$ was immobilized successfully. Equally important is that the fungal exudates bind specifically to the immobilized $\mathrm{Al}^{3+}$ and were retained during column washing. Finally, exudates could be eluted from NTA and IDA IMAC columns using $\mathrm{NH}_{4} \mathrm{OH}$ and the addition of ethanol to the elutor solution decreased the non-specific hydrophobic interactions. However, determining what impact, if any, the increased $\mathrm{pH}$ has on the exudates structure could not be determined at this stage. Based on the nature of these compounds and the mechanism of elution, structural degradation is unlikely. After comparison of many potential elutors, it was determined that 
the high $\mathrm{pH}$ and presence of both $\mathrm{NH}_{3}$ and $\mathrm{OH}^{-}$are required for optimal elution of the exudates due to the hard nature of $\mathrm{Al}^{3+}$ and these bases.

\subsubsection{Ligand comparison}

There appears to be little difference between IDA (tridentate) and NTA (tetradentate) for retention of $\mathrm{Al}^{3+}$ and isolation of the exudate complex. However, there does appear to be a minimal increase in exudate retention using the NTA ligand. It may be that this difference is due to column performance since the IDA columns are company manufactured while the NTA columns are packed in the lab or slight differences in the exudate batches. However, elution with $1 \mathrm{M} \mathrm{NH}_{4} \mathrm{OH}$ in $20 \%$ ethanol was able to successfully remove the $\mathrm{Al}^{3+}$ and exudate from the column in both cases. 


\section{RP and WAX HPLC of purified exudates}

\subsection{Introduction}

\subsubsection{Principle of reversed phase separation}

Reversed phase (RP) chromatography is one of the most common techniques used for the separation of larger biomolecules. RP chromatography separates compounds based on their relative hydrophobicity. Therefore, it is expected that more polar compounds will elute within the void volume and/or early in the separation. Larger compounds containing long aliphatic chains or aromatic rings will be bound to the column more strongly and elute later on. To elute molecules with a broad range of hydrophobicities, gradient elution can be utilized by increasing the organic content of the buffer throughout the chromatographic run. As the concentration of organic solvent increases, the analytes in the mobile phase are eluted in order of increasing hydrophobicity.

\subsubsection{Principle of weak anion exchange separation}

Weak anion exchange chromatography (WAX) is a common separation technique used for the separation of anions in solution. This form of ion exchange is termed "weak" because the anion exchanger used is a relatively weak base, such as a secondary or tertiary amine [25]. Analyte elution from a WAX column can be done using either a $\mathrm{pH}$ or salt gradient. By increasing the salt concentration in the mobile phase, the ionic strength of the environment is increased and elutes the analyte through a displacement mechanism. When using a $\mathrm{pH}$ gradient in WAX, the $\mathrm{pH}$ of the mobile phase is increased steadily to elute the anion by deprotonating the cationic functional group on the column. 


\subsubsection{Coupling of WAX and RP}

Two-dimensional separations (2D) have the advantage of increasing the peak capacity of a separation. The ideal circumstance is to have two dimensions that are orthogonal and hence separate the analyte on two independent mechanisms. Under these circumstances, the peak capacity of the $2 \mathrm{D}$ separation is the product of the peak capacities of the individual separations. 2D separations have often been used in the past and have the benefit of being able to further resolve a sample when the resolution of a single separation is too low. Not all separation techniques can readily be coupled together due to interferences with one dimension due to buffers from the other. Fortunately, WAX and RP use solvents/buffers that are compatible with each other. The organic buffers used in RP do not hinder the effectiveness of the WAX and, likewise, the salt gradient of a WAX will not interfere with the RP conditions. Also, it is believed the exudates contain acidic functional groups which would allow for effective separation using WAX. A further advantage to this combination is compatibility of solvents and ease of online coupling to the mass spectrometer. A WAX separation can be performed and the collected fractions loaded onto the RP column. The buffer salt can be rinsed out of the column and then the RP separation performed online with ESI-MS analysis. This method can be a time intensive process due to the salt rinsing and stepwise elution of the WAX separation, but the resultant increase in peak capacity is advantageous for analyzing complex mixtures.

\subsubsection{Rationale for research}

Despite the unknown structure of the fungal exudates, it is hypothesized that they complex the $\mathrm{Al}^{3+}$ through carboxylic acid groups and can therefore be separated by WAX. The fungal exudates most likely contain aliphatic and aromatic rings due to their 
absorbance and are hydrophobic in nature. Therefore, the exudates should be amenable to RP separation as the second dimension. Because WAX and RP are orthogonal, separating on different properties, it is possible to perform a two-dimensional separation utilizing these methods. Because the components in these exudate mixtures are smaller than most peptides and proteins, $\mathrm{RP}$ chromatography using $\mathrm{C}_{18}$ resins is preferred because it binds hydrophobic compounds more strongly than $\mathrm{C}_{8}$ due to its longer carbon chain. Therefore, even though the exudates may not be extremely hydrophobic, the binding to the column will be strong enough to allow a separation to be performed.

RP separations can be carried out under a variety of buffer conditions. In order to increase analyte retention and separation efficiency, optimization of buffer conditions such as $\mathrm{pH}$, ion pairing agents, and organic solvent concentration will help ensure the most favorable separation conditions. For example, RP separations are commonly performed at a $\mathrm{pH}$ of 3 for proteins and peptides. The acidic functional groups of the common amino acids have a $\mathrm{pK}_{\mathrm{a}}>3$ and will be protonated, adding no net charge to the molecule. However, these conditions are not practical for all analytes or RP applications. Furthermore, an increasing number of RP columns are becoming available which are stable over a wider $\mathrm{pH}$ range, allowing for separations under a variety of experimental conditions. Therefore, performing a RP separation at $\mathrm{pH} 11$ is advantageous because the IMAC purified exudates will remain uncomplexed at this high $\mathrm{pH}$ and stable RP stationary phases are available to accommodate this need.

WAX separates mixtures based on negative charges present in the molecules. Therefore, WAX can be used to separate the potentially negatively charged exudate. Once unbound exudates have been isolated, these isolates can be compared with exudate that 
have been bound with $\mathrm{Al}^{3+}$ on the MS. Ion signal shifts should be seen in the mass spectra due to Al-exudate complexes versus free exudate.

\subsection{Experimental}

Reversed phase separations were carried out using an Agilent 1100 series HPLC (Palo Alto, CA, USA) with a binary pump system and variable wavelength detector. Agilent Zorbax extended $\mathrm{C}_{18}$ columns $(2.1 \times 100 \mathrm{~mm}, 3.5 \mu \mathrm{m}$ beads, 80 A pores) were used (Palo Alto, CA, USA) for the RP-HPLC stationary phase. WAX separations were performed using PolyWax LP column from PolyLC Inc. (Columbia, MD, USA) and WAX Bakerbond SPE sep pack cartridges (J.T. Baker, Phillipsburg, NJ, USA). The aluminum concentration in RP eluent samples was determined using a model JY 2000 inductively coupled plasma-atomic emission spectrometer (ICP-AES) (Jobin Yvon, Edison, NJ, USA).

\subsubsection{Fate of $\mathrm{Al}^{3+}$ following IMAC elution}

An important element in this research was to determine if $\mathrm{Al}^{3+}$ was free in solution or complexed with the fungal exudates after IMAC isolation. Experimental results indicated the $\mathrm{Al}^{3+}$ was eluted from the IMAC column, therefore a series of experiments were performed to determine if an $\mathrm{Al}^{3+}$-exudate complex was present in the eluent.

The initial testing for $\mathrm{Al}^{3+}$ elution from the IMAC column was performed using eriochrome cyanine. Following elution of the exudate from the column with $\mathrm{NH}_{4} \mathrm{OH}, \mathrm{EC}$ was loaded on the column and did not change colors, indicating all metal cations were stripped from the column during the $\mathrm{NH}_{4} \mathrm{OH}$ elution. Additionally, the $\mathrm{EC}$ did not bind to the column and was washed through with the subsequent wash solution. In a control experiment it was shown that $\mathrm{EC}$ was immobilized and changed colors on an $\mathrm{Al}^{3+}$ activated

IMAC column. Once it was determined by qualitative EC and ICP-AES that the $\mathrm{Al}^{3+}$ 
eluted from the IMAC column with the pigment, the next step was to determine if the $\mathrm{Al}^{3+}$ was free in the solution or complexed with the pigment. To determine if $\mathrm{Al}^{3+}$ is free or complexed in the IMAC eluent solution, the IMAC eluent was separated by RP-HPLC and the fractions were analyzed by ICP-AES.

Initial RP separations were carried out at a $\mathrm{pH}$ of 3-4 to separate exudate that had not undergone IMAC isolation, thus determining if RP was a suitable technique. This $\mathrm{pH}$ was used to reduce the charge on the exudate compounds, rendering them more hydrophobic. The Agilent Zorbax extended $\mathrm{C}_{18}$ column was used (Palo Alto, CA, USA) for the RP-HPLC stationary phase. Buffer A was 5\% acetonitrile (HPLC Grade, Fisher Scientific, Pittsburgh, PA, USA), 1\% (v/v) acetic acid (Sigma-Aldrich, St. Louis, MO, USA), $0.05 \%(\mathrm{v} / \mathrm{v})$ heptafluorobutyric acid (Sigma-Aldrich) and buffer B was the same except with $100 \%$ acetonitrile. Fungal exudate was loaded onto the column via a $20 \mu \mathrm{L}$ injection loop and separated using a linear gradient of $0-100 \%$ acetonitrile in 30 minutes. The wavelength of detection varied due to environmental effects and the exudate batch used. Whenever conditions of the experiment changed, e.g. $\mathrm{pH}$ or origin of sample, the exudate was analyzed on the UV-Vis spectrophotometer to determine the best wavelength for detection. This wavelength was used in the HPLC detector and usually between 250$350 \mathrm{~nm}$ proved successful, depending on the particular experiment. Later installation of a diode array detector (DAD) permitted simultaneous detection with several wavelengths.

Following IMAC isolation, the eluted fraction was dried down in a Savant 110 speed vac (Albertville, MN, USA) to remove the IMAC solutions such as ethanol and ammonium hydroxide. This was done by setting the speed vac to "low" temperature and periodically checking the sample for complete removal of liquid. The remaining portion 
was redissolved in buffers appropriate for RP-HLPC separation (5\% acetonitrile, $1 \%$ acetic acid, $0.05 \%$ heptafluorobutyric acid) and sonicated to investigate the ratio of free $\mathrm{Al}^{3+}$ to complexed $\mathrm{Al}^{3+}$. Using this method, volatile solvents could be removed leaving only the compounds of interest behind. Another method employed to acidify IMAC fractions involved titration of the eluent to $\mathrm{pH} 3$ with acetic acid. This technique reduced both altering of the system and the potential for altering the state of the exudates caused by the drying process.

\subsubsection{RP-HPLC at low pH}

To determine changes in binding behavior due to $\mathrm{pH}$, RP separations were also carried out at $\mathrm{pHs} 1$ and 1.7. The separations at $\mathrm{pH} 1$ and 1.7 were performed to determine if the extreme $\mathrm{pH}$ would dissociate the $\mathrm{Al}^{3+}$ from the complex by protonating the acid moieties on the pigment. To evaluate this, the collected IMAC fractions were titrated to $\mathrm{pH}$ 1 with formic acid (>96\%) and to $\mathrm{pH} 1.7$ using glacial acetic acid, both provided by Sigma Aldrich (St. Louis, MO, USA). The end point $\mathrm{pH}$ for both titrations was determined using a pH meter. Polydivinyl benzene (PVB) polymeric beads (Hamilton, Reno, NV, USA) were used as the RP matrix for these $\mathrm{pHs}$ due to their improved stability at low $\mathrm{pH}$. A 15 cm length of 0.04 " ID Tefzel tubing was packed using the PDB beads at a pressure of 100 psi. A biocompatible filter assembly from Upchurch Scientific (Oak Harbor, WA, USA) was used as the frit.

\subsubsection{RP-HPLC at high $\mathrm{pH}$}

Higher $\mathrm{pH} 11$ separations were carried out so that changes in the matrix of the postIMAC fraction could be avoided and minimize system disturbances, such as altering any complexes due to a change in $\mathrm{pH}$. For these separations, 5\% acetonitrile, $1 \%$ ammonium 
hydroxide, and $0.05 \%$ tetrabutylammonium hydroxide (prepared from a Sigma-Aldrich $40 \%$ tetrabutylammonium hydroxide wt. solution in water) made up buffer A while buffer B was the same except with $90 \%$ acetonitrile. RP-HPLC was performed on the Agilent Zorbax extended $\mathrm{C}_{18}$ column (Palo Alto, CA, USA) using the same experimental conditions as previous HPLC separations. PVB beads (Hamilton, Reno, NV, USA) were packed at low pressure into emptied HiTrap ${ }^{\mathrm{TM}}$ cartridges $(1 \mathrm{~mL}$ volume, Amersham Pharmacia, Piscataway, NJ, USA) for low pressure bulk extractions.

It is hypothesized that if the exudates are eluted from the IMAC column at $\mathrm{pH} 11$, then they most likely do not complex the $\mathrm{Al}^{3+}$ under these conditions. RP-HPLC separations at $\mathrm{pH} 11$ are employed because of the benefit of solution consistency throughout the course of experiments. Ammonium hydroxide was used to adjust unseparated exudate to $\mathrm{pH} 11$ before loading on the RP column. IMAC fractions were loaded directly on the RP column without $\mathrm{pH}$ adjustment. Buffer A ran as an isocratic wash for 5 minutes before running the gradient elution to $100 \%$ buffer B from time $5-20$ minutes. An isocratic column wash step of $100 \%$ buffer B was performed for 5 minutes before dropping to $100 \%$ buffer A for a 5 minute re-equilibration. The RP fractions were collected every 10 minutes throughout the separation ( 3 fractions total) using the Agilent fraction collector. This scheme resulted in collection of a fraction containing any unretained, hydrophilic portion (free $\mathrm{Al}^{3+}$ ) and two increasingly hydrophobic fractions. Collected fractions were analyzed for $\mathrm{Al}^{3+}$ by ICP-AES analysis using appropriate standards. Copper was used as an internal standard in all standards and samples. Initially, ICP standards were prepared to match the organic content of the samples. It was soon evident that the recreated organic content in the blanks and standards were not an accurate 
representation of the organic background in the samples. In later preparation, samples were diluted to decrease organic concentration along with the standards. Minimal dilution was performed so aluminum concentrations were not decreased below the detection limits of the instrument. Another method used for ICP-AES sample preparation was the evaporation of the samples at $90^{\circ} \mathrm{C}$ for $2 \mathrm{hrs}$. The sample was brought back to its original volume with $15 \%$ nitric acid and $200 \mu \mathrm{L}$ hydrogen peroxide. Because of varying retention times due to separation conditions or sample properties, fractions collected for MS analysis were based on peaks rather than time. That is, fractions were collected based on the detection of a peak by the HPLC rather than during preset windows of time.

\subsubsection{WAX of unbound exudates}

Initially, salt was used to perform WAX elution. Buffer A consisted of $20 \mathrm{~m} M$ Tris and buffer B was $20 \mathrm{~m} M$ Tris, $1 M \mathrm{NaCl}$. Extractions were performed using the WAX SPE cartridge and solutions were pumped through the column with a syringe. These preliminary separations were performed to determine the effectiveness of WAX as an efficient separation technique for fungal exudate before optimizing a high separation efficiency technique using the HPLC. Flow was maintained at $0.5 \mathrm{~mL} / \mathrm{min}$ using a syringe pump. The cartridge was rinsed with A buffer, loaded with $1 \mathrm{~mL}$ fungal exudate, rinsed with A buffer, and then eluted with $100 \% \mathrm{~B}$ buffer. Initially, $1 M \mathrm{NaCl}$ was used as the elution B buffer but later attempts utilized the same method except B buffers attempted were $1 M$ solutions of sodium acetate, Tris, citrate, sodium phosphate, and potassium phosphate. 


\subsection{Results and Discussion}

\subsubsection{Fate of $\mathrm{Al}^{3+}$ following IMAC elution}

Attempts to analyze the $\mathrm{Al}^{3+}$ purified pigment exudates directly by ESI-MS proved to be unsuccessful. ESI-MS spectra of these samples contained no reproducible peaks as the spectra essentially looked like background spectra with greater signal intensity. The inability to distinguish discrete ion signals was attributed to the complexity of the samples and the limited loading capacity and resolving power of the ion-trap MS. If multiple exudate ligands can bind to the $\mathrm{Al}^{3+}$, the presence of complexes in solution will greatly increase the complexity of the samples. Furthermore, if the binding of the exudate ligands to the $\mathrm{Al}^{3+}$ is very strong, then the fragmentation in the CID spectra will be reduced, decreasing the likelihood of obtaining interpretable CID spectra. For these reasons, it is essential to determine whether the isolated fungal exudates are complexed or uncomplexed with the $\mathrm{Al}^{3+}$ following IMAC elution. Not only do we want to know the mechanism of IMAC elution, but determination of the complexation state of the pigment is required for its characterization. Reversed phase separations of non-IMAC extracted exudate at $\mathrm{pH} 3$ demonstrated the utility of the technique for the separation of the pigments as shown in Figure 4.1. 


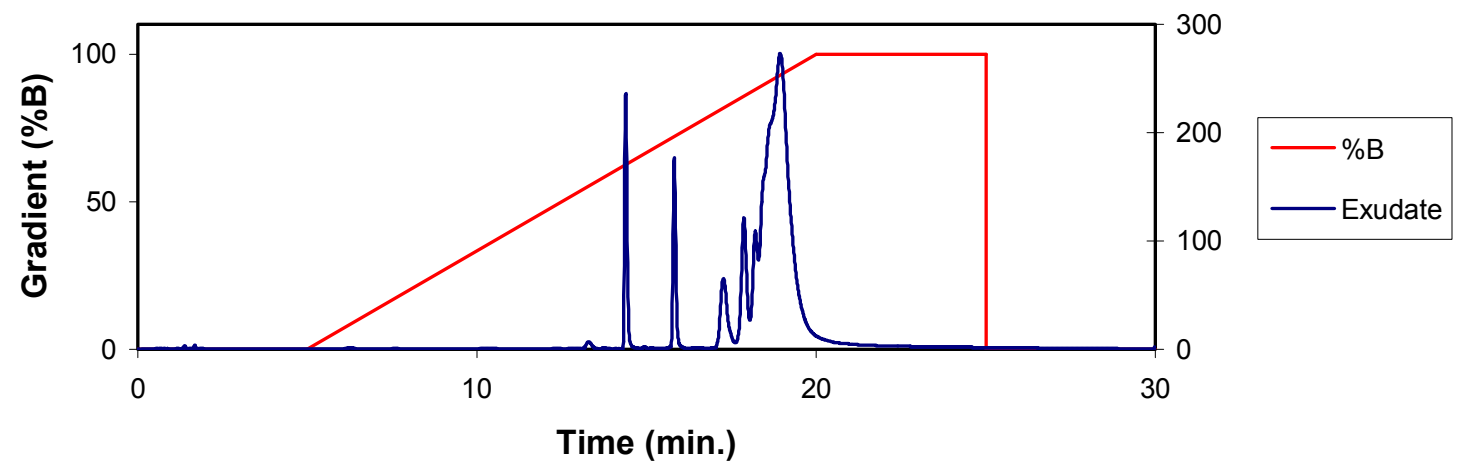

Figure 4.1-RP separation of unpurified fungal exudate at $\mathrm{pH} 3$. Flow rate $=0.2 \mathrm{~mL} / \mathrm{min}$, Zorbax ext. $\mathrm{C}_{18}$ column, $\lambda=350 \mathrm{~nm}$.

\subsubsection{RP-HPLC at low pH}

The RP separations of the fungal exudates purified by IMAC were ultimately unsuccessful at $\mathrm{pH}$ 3. Therefore, the next objective was to find conditions that would dissociate the ligand and $\mathrm{Al}^{3+}$ complexes. A first attempt at complex dissociation was performed by decreasing the $\mathrm{pH}$ to 1.7 and 1 to: 1) to competitively dissociate $\mathrm{Al}^{3+}$ from any potential complexes by facilitating a proton rich environment and, therefore, produce a molecule with a lower overall positive charge (more hydrophobic) and, 2) provide an environment where negatively charged sites on the exudates would be protonated and reduce it's polarity if no complexes were present. Two techniques were used to remove basic IMAC solutions and lower the $\mathrm{pH}$ of the IMAC eluent solution containing the exudates. The first technique dried IMAC fractions at different rates and to different degrees in the speed vac to remove volatile components. Attempts with varying concentrations of acetonitrile and/or acetic acid to redissolve these dried samples were unsuccessful. Some exudate was observed to go back into solution but most remained as an insoluble precipitate or adsorbed to the tube walls. The concentration of the redissolved exudate was so low that the loss of potentially vital components resulted in no interpretable 
data using this method. Cumming et al. observed this same behavior under a variety of conditions when drying and attempting to resuspend fungal exudates. IMAC fractions were also prepared for RP by direct acidification to $\mathrm{pH} 3$ using acetic acid. The exudate in the IMAC fraction remained intact and in solution following acidification. However, when loaded on the RP column, the IMAC fraction was unretained and eluted in the void volume as shown in Figure 4.2. It was surmised that the lack of retention of the IMAC purified exudates could be caused by complexation of the $\mathrm{Al}^{3+}$ by the ligands, resulting in a more hydrophilic moiety. Previously it was determined that the $\mathrm{Al}^{3+}$ was removed from the IMAC column during the elution, so $\mathrm{Al}^{3+}$ is present in the IMAC eluent solution. Complex formation would render the pigment exudates more hydrophilic, and decrease their retention on the column. Ultimately, lowering the $\mathrm{pH}$ of the IMAC fractions from 11 to 3 led to no binding on the RP column and negated the usefulness of RP under the lower $\mathrm{pH}$ conditions.

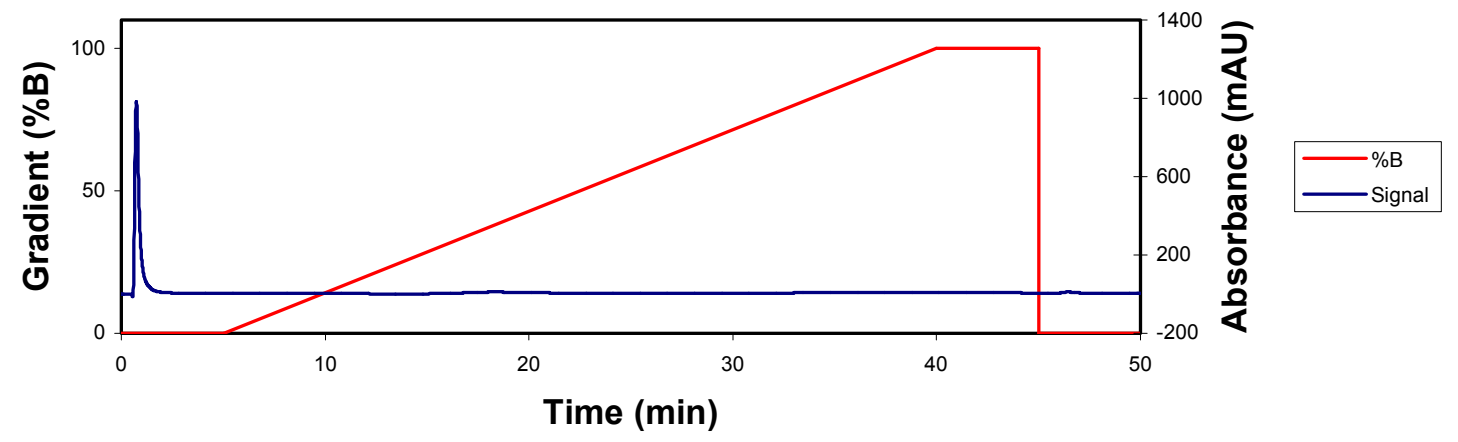

Figure 4.2-RP separation of IMAC isolated exudate fraction titrated to $\mathrm{pH} 3$ with acetic acid. Flow rate $=0.2 \mathrm{~mL} / \mathrm{min}$, Zorbax ext. $\mathrm{C}_{18}$ column, $\lambda=250 \mathrm{~nm}$.

After the RP-separation of the exudate pigments was unsuccessful at $\mathrm{pH} 3$ the $\mathrm{pH}$ was further decreased to $\mathrm{pH} 1$ and 1.7. It was anticipated that further decrease in the $\mathrm{pH}$ would protonate the acidic moieties that were believed to complex $\mathrm{Al}^{3+}$. Therefore, the 
$\mathrm{Al}^{3+}$ exudate complex would be dissociated and the acidic sites protonated to help increase the exudates hydrophobicity on the RP column. However, as seen in Figure 4.3 and 4.4, the exudates were unretained and eluted in the void volume for the RP separations at $\mathrm{pH} 1$ and 1.7 , yielding nearly the same results as $\mathrm{pH} 3$. This method of $\mathrm{pH}$ reduction also failed because the decrease in $\mathrm{pH}$ would not fully redissolve the pigment exudates from the IMAC extracted fraction, despite the use of different acids and lower pHs.

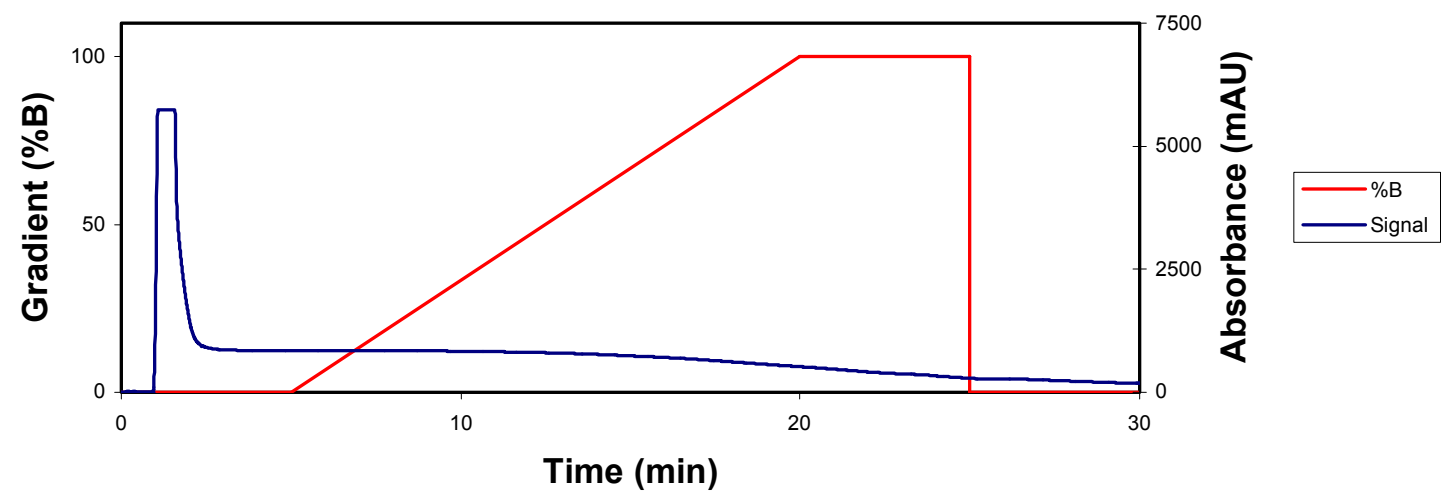

Figure 4.3-RP separation of IMAC isolated fraction performed at $\mathrm{pH} 1$. Flow rate $=0.2$ $\mathrm{mL} / \mathrm{min}$, POROS RP column, $\lambda=250 \mathrm{~nm}$.

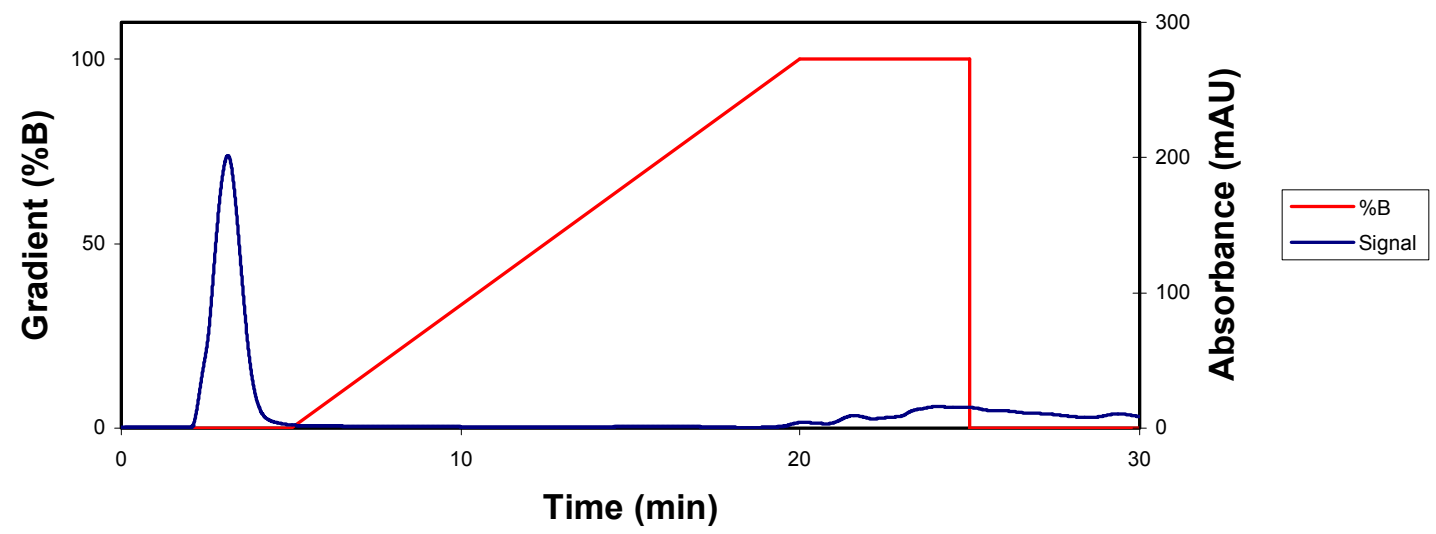

Figure 4.4-RP separation of IMAC isolated fraction performed at $\mathrm{pH}$ 1.7. Flow rate $=0.2$ $\mathrm{mL} / \mathrm{min}$, POROS RP column, $\lambda=250 \mathrm{~nm}$. 


\subsubsection{RP-HPLC at high pH}

After RP separation of the IMAC purified exudates was unsuccessful at low $\mathrm{pH}$, the separation was performed at $\mathrm{pH} 11$. The RP separation was performed at $\mathrm{pH} 11$ because this was the $\mathrm{pH}$ of the IMAC elutor solution and eluent. The objective was to determine if the exudates and $\mathrm{Al}^{3+}$ were unbound or complexed after elution from the IMAC cartridge. Elution of the bound metal ion and analyte has been termed metal ion transfer [11]. It was hypothesized that because the $\mathrm{Al}^{3+}$ was stripped off the IMAC column at this $\mathrm{pH}$ that it was also possible the exudate ligands were unbound at this $\mathrm{pH}$. Performing RP separation at $\mathrm{pH} 11$ permitted analysis of the sample in the same state it left the IMAC column in. If any exudate complexes are present, they will remain intact during the $\mathrm{RP}$ at $\mathrm{pH} 11$ because basic conditions were also used for IMAC elution. First the bulk, non-IMAC extracted, pigments were separated by RP chromatography at pH 11 .

As shown in Figures 4.5A \& B, the RP gradient separation of the bulk exudate pigments was successful as the exudates were strongly retained and many peaks were resolved. The two traces from the chromatogram correspond to the absorbance at $\lambda=350$ $\mathrm{nm}$ and $\lambda=250 \mathrm{~nm}$, which allows for differentiation of the fungal exudates from media components. The large peak present at 15.3 minutes in Figure $4.5 \mathrm{~B}$ was formed by the liquid media that the exudates were grown in. The media components were identified by matching the retention times and spectral characteristics of similar peaks with a chromatogram of the liquid growth media blank.

In Figure 4.6, the separation of the IMAC purified exudates by gradient chromatography is shown with detection at $350 \mathrm{~nm}$. Therefore even in the presence of $\mathrm{Al}^{3+}$ the exudate pigments are strongly retained at $\mathrm{pH} 11$, which indicates that the 
pigment exudates are more hydrophobic at this $\mathrm{pH}$. The increase in hydrophobicity is most likely caused by dissociation of the $\mathrm{Al}^{3+}$ from exudate pigments. However, the $\mathrm{Al}^{3+}$ concentration in each of the fractions must be determined for a more definitive answer. If the $\mathrm{Al}^{3+}$ and exudate pigments are uncomplexed, then the free $\mathrm{Al}^{3+}$ will be very hydrophilic and will elute in the void volume while the exudate pigments will elute without $\mathrm{Al}^{3+}$ in latter fractions. Additionally, when the chromatograms of the bulk and post-IMAC exudates are compared, it is seen that the IMAC isolation is useful for simplifying the mixture. When an IMAC isolated fraction is separated by RP at $\mathrm{pH} 11$, the components of the liquid media are removed along with exudates that are not $\mathrm{Al}^{3+}$ binding. Removal of the non- $\mathrm{Al}^{3+}$ binding components can be seen by comparing Figure 4.6 to Figure 4.5A. Various peaks are consistent between these chromatograms, which would be expected, while fewer peaks are present in Figure 4.6. Furthermore, the absorbance trace at $\lambda=250 \mathrm{~nm}$ shows no liquid media components are present after IMAC purification. Due to the complexity of the exudate mixture, many components were not fully separated by RP. 


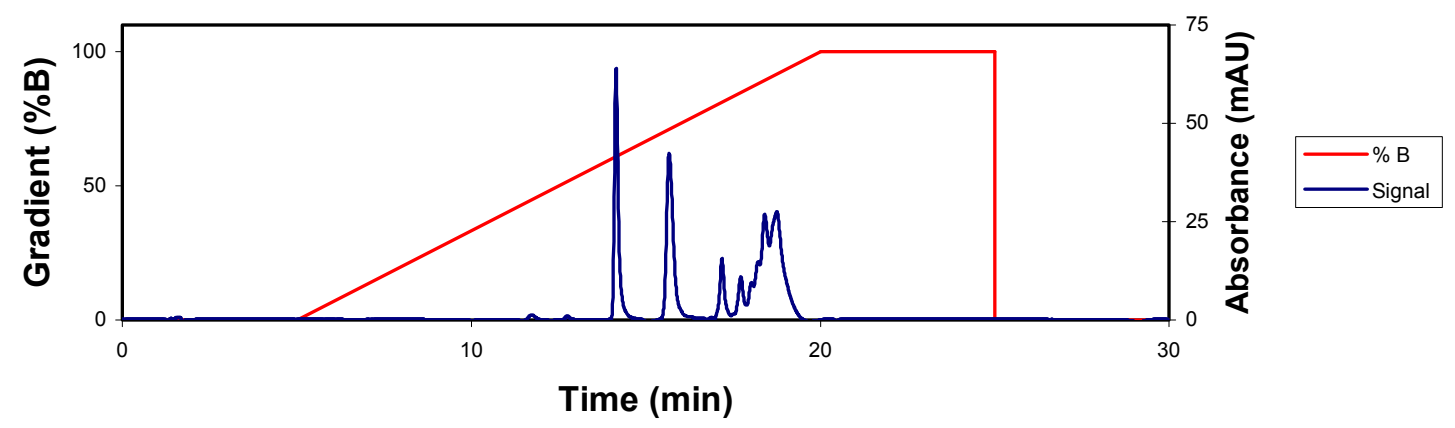

Figure 4.5A-RP separation of fungal exudate at $\mathrm{pH} 11$. Flow rate $=0.2 \mathrm{~mL} / \mathrm{min}$. Zorbax ext. $\mathrm{C}_{18}$ column, $\lambda=350 \mathrm{~nm}$. Chromatogram contains peaks representing fungal exudate.

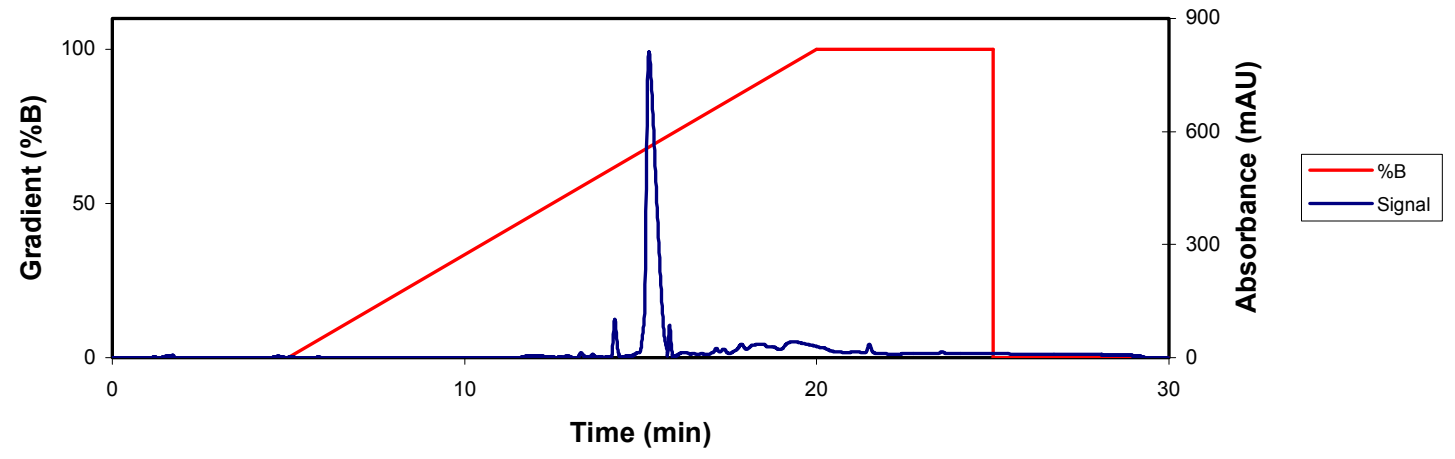

Figure 4.5B-RP separation of fungal exudate at $\mathrm{pH} 11$. Flow rate $=0.2 \mathrm{~mL} / \mathrm{min}$. Zorbax ext. $\mathrm{C}_{18}$ column, $\lambda=250 \mathrm{~nm}$. Chromatogram contains peak representing liquid media.

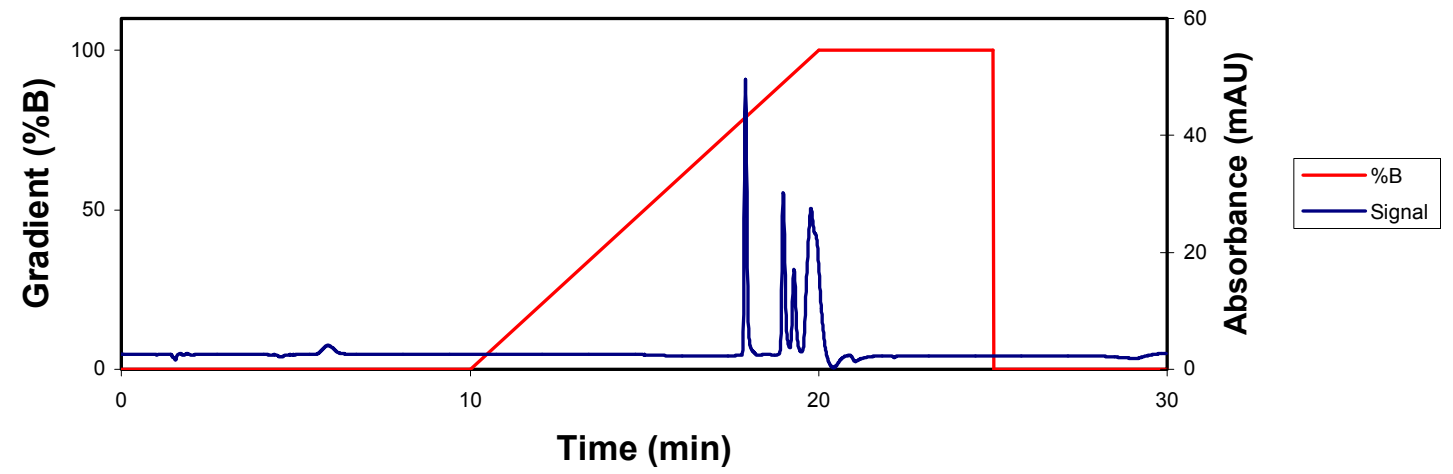

Figure 4.6-RP separation of IMAC isolated exudate at $\mathrm{pH} 11$. Flow rate $=0.2 \mathrm{~mL} / \mathrm{min}$. Zorbax ext. $\mathrm{C}_{18}$ column, $\lambda=350 \mathrm{~nm}$. 
ICP-AES analysis of fractions collected from the $\mathrm{pH} 11 \mathrm{RP}$ separations revealed a high concentration of $\mathrm{Al}^{3+}$ in the void volume, and only trace levels of $\mathrm{Al}^{3+}$ were detected in the latter more hydrophobic fractions collected (Table 4.1). This observation suggests that after IMAC isolation, the $\mathrm{Al}^{3+}$ and exudates dissociate and remain free in solution. This idea is supported by the concentration of $\mathrm{Al}^{3+}$ in the hydrophobic fractions were not significantly different from the background, indicating that only trace levels of $\mathrm{Al}^{3+}$ are present with the exudates. This trend was also observed when bulk RP separations were performed on low pressure columns and the eluent analyzed. Therefore, ICP-AES analysis of RP purified samples indicate that $\mathrm{Al}^{3+}$ does dissociate from the exudates during isolation. Most importantly, a method for extraction and isolation of $\mathrm{Al}^{3+}$ free pigment exudates has been developed.

\begin{tabular}{cccc}
\hline RP Fraction & $\mathbf{A l}^{\mathbf{3 +}} \mathbf{( \% )}$ & $\mathbf{\% B}$ & Time (min.) \\
\hline Flow Through & 94 & 5 & 0 \\
1 & 5 & $5-11$ & $0-10$ \\
2 & 1 & $11-100$ & $10-30$ \\
\hline
\end{tabular}

Table 4.1-Distribution of $\mathrm{Al}^{3+}$ among $\mathrm{pH}=11 \mathrm{RP}$ fractions following IMAC-RP purification. RP fractions analyzed using ICP-AES, including appropriate $\mathrm{Al}^{3+}$ standards and $\mathrm{Cu}^{2+}$ internal standards. Fractions collected between 10-20 and 20-30 minutes were combined due to trace levels of $\mathrm{Al}^{3+}$ detected in both fractions.

\subsubsection{WAX of unbound exudates}

Ultimately, WAX proved impractical for the separation of fungal exudates, and, in the end, a 2D separation was not needed due to the sequential use of IMAC and RP. Originally, it was believed that the exudates bound strongly to the WAX column but this turned out not to be the case. Instead, it was determined that the exudates were fouling the column, an event relatively common among acidic organic compounds [27]. Column fouling occurs when the analyte becomes irreversibly fixed to the column. Analyte 
precipitation, irreversible retention, or loss of resin stability are common causes of fouling which leads to poor recovery, increased back pressure, and band broadening, along with other detrimental effects. No attempts to clean or reequilibrate the column were successful as the exudate plug could clearly be seen on the sep pack columns bound at the top of the column.

\subsection{Conclusions}

\subsubsection{Advantage of multiple separations}

$\mathrm{RP}$ allows for the separation of free $\mathrm{Al}^{3+}$ and $\mathrm{Al}^{3+}$-specific exudates which is of great value. At $\mathrm{pH} 11, \mathrm{RP}$ also proves to be an effective and convenient method to separate bulk and IMAC purified fungal exudates. Obtaining an exudate sample which contains only the $\mathrm{Al}^{3+}$ binding constituents of the original mixture makes subsequent analysis much easier. Although 2D separations proved to be impractical for the separation of the IMAC purified exudates, it has been established that a $2 \mathrm{D}$ technique is not necessary. IMAC and $\mathrm{RP}$ have the ability to isolate and purify the $\mathrm{Al}^{3+}$ binding exudates efficiently and provide a suitable sample for MS analysis. 


\section{Mass Spectrometry}

\subsection{Introduction}

\subsubsection{Principle of ESI-Ion Trap MS}

Electrospray ionization mass spectrometry (ESI-MS) has become a common ionization technique for mass spectrometric analysis of biomolecules, primarily because it is the method of choice for generation of molecular ions from solution. ESI is easily coupled with liquid chromatography for online separations, it is a soft ionization source capable of producing multiply charged species, and many of the mass analyzers ESI is used with are capable of performing collision-induced dissociation (CID) to provide further structural information. The formation of multiply charged species permits the analysis of larger biomolecules, by allowing them to be monitored by mass analyzers with a lower mass range by decreasing their $\mathrm{m} / \mathrm{z}$. Also, higher charge states generally provide more information after CID due to the greater number of charged functional groups available for dissociation. Figure 5.1 shows a general diagram of an ESI source coupled with an ion trap mass analyzer.

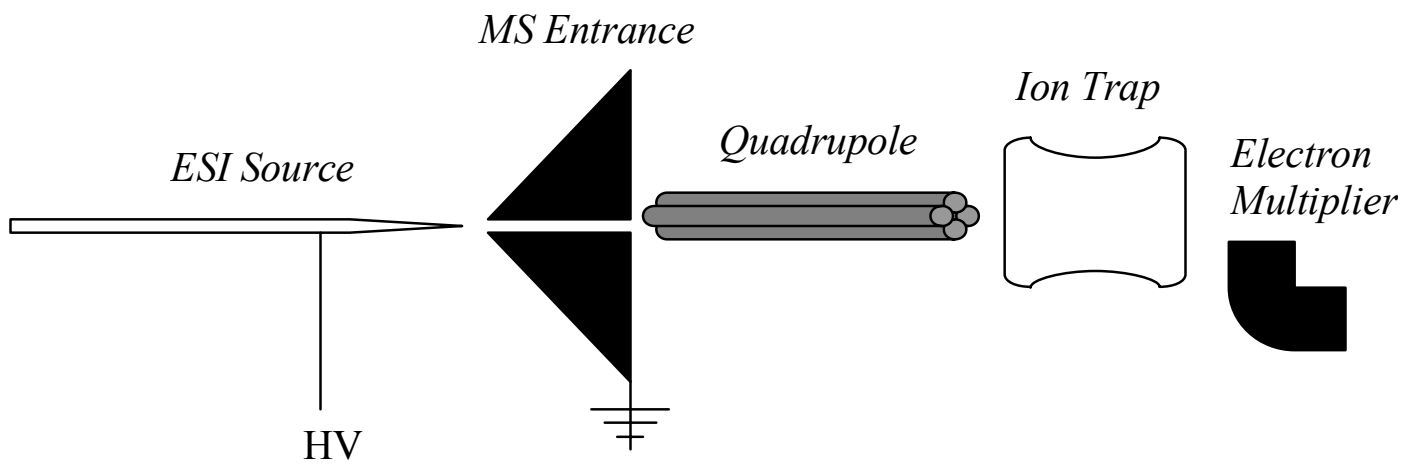

Figure 5.1-Basic schematic of an ESI-MS with Ion Trap. 
In the past, fast atom bombardment ionization (FAB) sources, played a larger role in biomolecule analysis [25]. More recently, matrix-assisted laser desorption ionization (MALDI) and ESI were developed as more favorable ionization techniques. Although MALDI is still commonly used, both FAB and MALDI have disadvantages which lead to the increasing favor for ESI. Both FAB and MALDI require the sample to be dissolved in a matrix and are not easily coupled with HPLC. In FAB, the analyte-doped matrix crystal is then hit with a particle beam of neutral atoms to produce ions. Alternatively, the matrix crystal is irradiated with a laser for MALDI to achieve ionization [25]. ESI can be used for ionization of a sample mixture or for direct analysis of samples separated on-line. The ESI interfaces used for bulk sample introduction and online liquid-phase separations are very similar. The difference between these two techniques is that direct infusion of a mixture introduces the sample at a constant flow rate to analyze all components in the mixture simultaneously while online liquid-phase separations are used to reduce the number of components introduced into the MS per unit time. MALDI gives higher sensitivity than $\mathrm{FAB}$, but does not form multiply charged species, as ESI is capable of doing. Because MALDI is a pulsed ionization source, it couples well with time-of-flight (TOF) analyzers to identify ions within a matrix spot, and TOF-TOF instruments allow for tandem mass spectral analysis. Furthermore, improvements in coupling MALDI with trap mass analyzers have helped increase the sensitivity by adding an ion accumulation period. Additionally, ESI also has the ability to easily switch from positive to negative ion modes, promotes high sensitivity, and produces multiply charged species, making it particularly well suited for the analysis of large biomolecules from solution. 
ESI is compatible with the operational requirements of several mass analyzers. As with ionization sources, different mass analyzers have different performance characteristics that determine their suitability for a particular application. The ion trap is a good choice because it is capable of achieving high sensitivity, performing tandem-MS, and is compact. Ion traps also compliment ESI sources well. The ability of the ion trap to accumulate ions in the trap increased the duty cycle. Ions can be selected and accumulated before being ejected from the trap. Fourier transform instruments (FT-MS) are also very sensitive due to their trapping abilities while triple quad instruments cannot collect as large of an ion representation from the sample (lower duty cycle) because it is a continuous analyzer. Therefore, the triple quad instruments are not as high sensitivity as the trapping FT-MS.

Trap analyzers are said to be tandem in time which refers to the traps ability to dissociate and analyze the parent ion within the same volume. This process differs from the triple quad mass analyzer that is tandem in space because the ion dissociation and analysis are performed sequentially in different spatial areas. Both triple quad and ion trap instruments can be used to perform CID of biomolecules but by differing means. Ion traps have the ability to accumulate ions in the trap and eject a desired $\mathrm{m} / \mathrm{z}$ while a quadrupole isolates ions during flight by selectively focusing ions of interest. Ions can be collected and selected from the group, resulting in a higher duty cycle, which increases the sensitivity of the instrument. A triple quad instrument cannot accumulate ions over time since it is a continuous scanning mass analyzer. Continuous scanning instruments, such as quadrupoles, have a relatively low duty cycle. This means only a small representation of the total ions present in the sample are detected per unit time. 
The ion trap is not without its limitations. There is an upper and lower $\mathrm{m} / \mathrm{z}$ cut off and it is vulnerable to space charge effects. Due to excitation energies within the trap, larger and smaller ions are not stable in the trap and cannot be isolated. The triple quadrupole gives the ability to view a lower $\mathrm{m} / \mathrm{z}$ range. This is especially important in CID where the lower $\mathrm{m} / \mathrm{z}$ fragments are unstable due to the excitation energy. In a TOF instrument, all but the smallest fragments can be analyzed, because the fragment ion trajectories can be maintained over the entire $\mathrm{m} / \mathrm{z}$ range. TOF mass analyzers theoretically have no upper $\mathrm{m} / \mathrm{z}$ limit; however, the low kinetic energy of high $\mathrm{m} / \mathrm{z}$ ions prevents their detection, which creates a practical upper limit to the $\mathrm{m} / \mathrm{z}$ range. When an ion enters the TOF, the detector measures the time it takes the ion to traverse the flight path. This flight time depends on the ion's kinetic energy which is a function of the ion's $\mathrm{m} / \mathrm{z}$.

Many MS systems are used in biomolecular analysis. Depending on the particular needs and funds available, other options such as MALDI-TOF or ESI-triple quad may be better suited. Because the ion trap has high sensitivity, CID capability, adequate resolving power, and the ability to work within an $\mathrm{m} / \mathrm{z}$ range that compliments ESI, it is a suitable system for analysis of the fungal exudate pigments.

\subsubsection{Rationale for Research}

ESI-MS is a well-established analysis technique for polymers, peptides and proteins, and smaller polar compounds [25]. The same characteristics that make MS useful for these more common biomolecules are also required for the biologically produced fungal exudates. As previously stated, ESI-MS is excellent for the analysis of dilute charged species from solution and is readily combined with liquid phase separations. These attributes make ESI-MS an excellent choice for this research. Using ESI-MS, the mass, 
charge state, and complexity of the exudate pigment samples can be determined. Furthermore, CID spectra can be used for further structural characterization. Additionally, the instrument can be easily switched between the negative and positive ion modes for analysis of the pigment exudates.

\subsection{Experimental}

\subsubsection{Direct infusion of exudate}

Initially, ESI-MS analysis of exudates was performed using the LCQ Deca XP (Thermo Finnigan, West Palm Beach, FL, USA), a quadrupole ion trap instrument coupled with an electrospray ionization source. The software utilizes Xcaliber and Tune Plus programs for controlling the instrument. A high resolution CCD camera (TM-200, JAI Pulnix, Inc., Sunnyvale, CA, USA) with an Optem Zoom 70XL lense (Fairport, NY, USA) was used to observe the capillary tip and look for clogs or imperfections. Capillary spray tips were pulled with a P-2000 laser puller (Sutter Instrument, Novato, CA, USA). Later, the Thermo Electron linear trapping quadrupole-fourier transform-mass spectrometer (LTQ-FT-MS) instrument (West Palm Beach, FL, USA) was used for exudate characterization. The instrument was controlled using Xcaliber and LCQ Tune software. The triple quadrupole instrument, TSQ-700 MS (Thermo Finnigan, West Palm Beach, FL, USA) was also used briefly during the course of the exudate analysis.

With this instrument, direct infusion is achieved by introducing the exudate mixture with a syringe pump. The exudate was first diluted 10:1 in methanol to improve desolvation. To utilize this sample introduction method, a flow rate of $\sim 0.10 \mu \mathrm{L} / \mathrm{min}$ was typically used and other conditions, such as the electrospray voltage potential $(1.2-1.7 \mathrm{kV})$, were varied and adjusted "on the fly" in order to optimize spray and signal. Analyte 
solution was introduced through a $75 \mu \mathrm{m}$ silica capillary with a $10 \mu \mathrm{m}$ tip. Analysis of the untreated exudate was performed in positive ion mode since the $\mathrm{pH}$ of the exudate is between 3 and 4 . In order to further examine the bulk exudate, aliquots were treated with $\mathrm{NH}_{4} \mathrm{OH}$ to raise the $\mathrm{pH}$ to 11 for negative ion mode analysis.

ESI-MS analysis was also utilized to examine IMAC purified exudates. Bulk exudates were isolated using the IMAC method described in Section 3.2.1. These isolated pigments were sprayed into the MS using direct infusion in both positive and negative ion modes. Instrumental conditions for this analysis were consistent with those used for the direct infusion of bulk exudate mixtures to obtain consistent spray. The flow rate was held at $\sim 0.10 \mu \mathrm{L} / \mathrm{min}$ and the electrospray voltage $(1.2-1.7 \mathrm{kV})$ were adjusted to optimize spray and signal. A $75 \mu \mathrm{m}$ silica capillary with a $10 \mu \mathrm{m}$ tip was used for analyte introduction.

Before each analysis, a standard was analyzed to make sure the instrument was operating properly or needed tuned. A $1.3 \mathrm{pg} / \mu \mathrm{L}$ angiotensin solution $(500 \mu \mathrm{L}$ methanol, $499 \mu \mathrm{L}$ dI water, $1 \mu \mathrm{L}$ acetic acid, and $2.6 \mu \mathrm{L} 500 \mathrm{pmol} / \mu \mathrm{L}$ angiotensin I human (SigmaAldrich, St. Louis, MO, USA)) was used as the standard in positive ion mode. The standard used in the negative ion mode was $0.65 \mathrm{pg} / \mu \mathrm{L}$ insulin chain A oxidized $(500 \mu \mathrm{L}$ methanol, $499 \mu \mathrm{L}$ dI water, $1 \mu \mathrm{L}$ ammonium hydroxide, and $2.6 \mu \mathrm{L}$ of $250 \mathrm{pmol} / \mu \mathrm{L}$ insulin chain A oxidized ammonium salt (Sigma-Aldrich, St. Louis, MO, USA)). The instrument parameters were acceptable when standards had an intensity exceeding $10^{8}$.

\subsubsection{Removal of free $\mathrm{Al}^{3+}$ from the IMAC eluent with RP extraction}

For removal of the $\mathrm{Al}^{3+}$ prior to MS analysis a low pressure, an RP extraction cartridge was used to generate a larger amount and volume of sample. The use of the larger RP extraction cartridge here is in contrast to the initial analytical separation of the 
$\mathrm{Al}^{3+}$ and exudate pigments described in Chapter 4. The IMAC column was washed with 4 $\mathrm{mL}$ wash solution, loaded with $2 \mathrm{~mL} 10 \mathrm{mM} \mathrm{AlCl}$, $4 \mathrm{~mL}$ wash solution, $2 \mathrm{~mL}$ fungal exudate loaded, $4 \mathrm{~mL}$ wash solution, and then eluted with $4 \mathrm{mLs} 1 M \mathrm{NH}_{4} \mathrm{OH}$. The eluted fraction was collected and loaded onto the RP column using a syringe pump and flow of 1 $\mathrm{mL} / \mathrm{min}$. The RP column contained POROS beads (Applied Biosystems, Foster City, CA, USA) that were used due to their greater stability over a wide $\mathrm{pH}$ range. The POROS beads were packed into an empty, $1 \mathrm{~mL}$ volume $\operatorname{HiTrap}^{\mathrm{TM}}$ cartridge (Amersham Pharmacia, Piscataway, NJ, USA) cartridge. The cartridge was packed by pouring a small amount of the POROS bead material into the cartridge, manually dry packing it with a fitting plunger to eliminate dead space, then repeating in small increments for even distribution until the cartridge was full, fritted, and capped. Buffer A was $5 \%$ acetonitrile, $0.5 \%$ ammonium hydroxide, $0.05 \%$ tetrabutylammonium hydroxide and buffer B was the same except with $90 \%$ acetonitrile. The RP separation was performed at a $\mathrm{pH}$ of 11 to change the conditions of the IMAC fraction as little as possible. After RP column equilibration, $1 \mathrm{~mL}$ of the IMAC purified pigment was loaded to the RP cartridge and $4 \mathrm{~mL}$ buffer A passed over the column to wash unbound compounds through the column. A stepwise elution was performed by eluting the retained species with $4 \mathrm{~mL}$ of $100 \%$ buffer $\mathrm{B}$ at $1 \mathrm{~mL} / \mathrm{min}$ which were collected. This sample was now free of $\mathrm{Al}^{3+}$ non-specific compounds as well as any excess $\mathrm{Al}^{3+}$ from the IMAC isolation.

\subsubsection{Assaying for $\mathrm{Al}^{3+}$ Complexation}

For further analysis, fungal exudate samples that were isolated by IMAC and the $\mathrm{Al}^{3+}$ removed were treated to analyze them in solutions of different $\mathrm{pHs}$ and in the presence of $\mathrm{Al}^{3+}$. Four samples were prepared from the same IMAC-RP purification. The first 
sample was an aliquot of the IMAC-RP purified eluent with no treatment. The second was left at $\mathrm{pH} 11$ and had $\mathrm{Al}\left(\mathrm{NO}_{3}\right)_{3}$ added to a final concentration of $0.1 \mathrm{ppm}$. The third sample consisted of an aliquot of the IMAC-RP fraction adjusted to $\mathrm{pH} 3$ with acetic acid. Finally, an IMAC-RP purified fraction was adjusted to $\mathrm{pH} 3$ and spiked to a concentration of 0.1 ppm $\mathrm{Al}\left(\mathrm{NO}_{3}\right)_{3}$. All samples were analyzed in positive and negative ion modes to look for peak shifts resulting from the addition of $\mathrm{Al}^{3+}$ and any complexation resulting from this $\mathrm{Al}^{3+}$ addition. Mass spectrometric analysis was performed in the same manner as described for previous direct infusion trials.

\subsection{Results and Discussion}

\subsubsection{Direct infusion of exudate}

Optimizing spray conditions for the MS was critical to ensure proper ionization and an accurate representation of the ion population. A $75 \mu \mathrm{m}$ ID silica capillary pulled to a spray tip ID of $\sim 10 \mu \mathrm{m}$ to provide small solvent droplets at the tip and, therefore, assist in producing sufficient spray. Adjusting the syringe pump on the LTQ-FT-MS to provide a flow rate of $\sim 0.10 \mu \mathrm{L} / \mathrm{min}$. and an electrospray voltage between $1.5-1.7 \mathrm{kV}$ was successful in providing efficient ionization. At times, the formation of crystals was observed (using the camera) on the spray tip and this formation was accompanied by signal degradation. This behavior was observed for all samples, including dilution of sample in methanol, larger spray tips, and varying flow rates did not eliminate crystal formation. The only effective remedy to this problem was to monitor the source and change the tip as warranted.

Analysis of non-purified exudates by MS provided initial insight into the fungal exudates and demonstrated the complexity of the fungal exudates. Figure 5.2 contains a 
typical spectrum of untreated fungal exudates using direct infusion analysis performed in positive ion mode. This spectrum resembles typical ESI background spectra, as no stable discrete ion signals were observed. A plausible explanation for the lack of observable peaks is that the sample complexity is very high and this level of complexity exceeds the loading capacity and resolving power of the ion trap MS. The loading capacity of the trap is limited by space charge effects, which degrade the resolving power of the MS when it is overloaded. The limited loading capacity and resolving power of the trap MS can prevent observation of a stable ion signal for extremely complex samples, because not enough of any one sample ion accumulates to levels that exceed the detection limit. Not only is the mixture of exudates present, but the non-aluminum binding compounds and the media are still present. The media contains sugars and nutrients that produce interfering contaminant signals in the spectra. A similar pattern is observed in Figure 5.3 which contains a spectrum acquired from a direct infusion analysis of bulk exudates performed in negative ion mode. Both positive and negative ion modes result in spectra too complicated to gather useful information from.

Because the exudates are not isolated or separated in these samples, many of the components from the media and components that are not $\mathrm{Al}^{3+}$ binding exuded by the fungus are still present. Large numbers of species and limited loading capacity of the trap prevent further analysis without reducing sample complexity. With no prior isolation, the number of ions entering the trap from compounds of no interest would lead to strong space charge effects and poor resolution. These spectra demonstrate that further analysis requires sample purification to reduce the sample complexity and permit analysis of the $\mathrm{Al}^{3+}$ binding components in the sample. We chose $\mathrm{Al}^{3+}$-IMAC isolation because it removes the 
media and the exudate components that do not bind $\mathrm{Al}^{3+}$. The IMAC isolated fungal exudates are fractionated by RP chromatography so the different $\mathrm{Al}^{3+}$ specific components can be analyzed by the MS. By performing the IMAC and RP in series, the $\mathrm{Al}^{3+}$ specific exudates are isolated and free $\mathrm{Al}^{3+}$ cations can be separated out, leaving the uncomplexed $\mathrm{Al}^{3+}$ specific exudates in the sample.

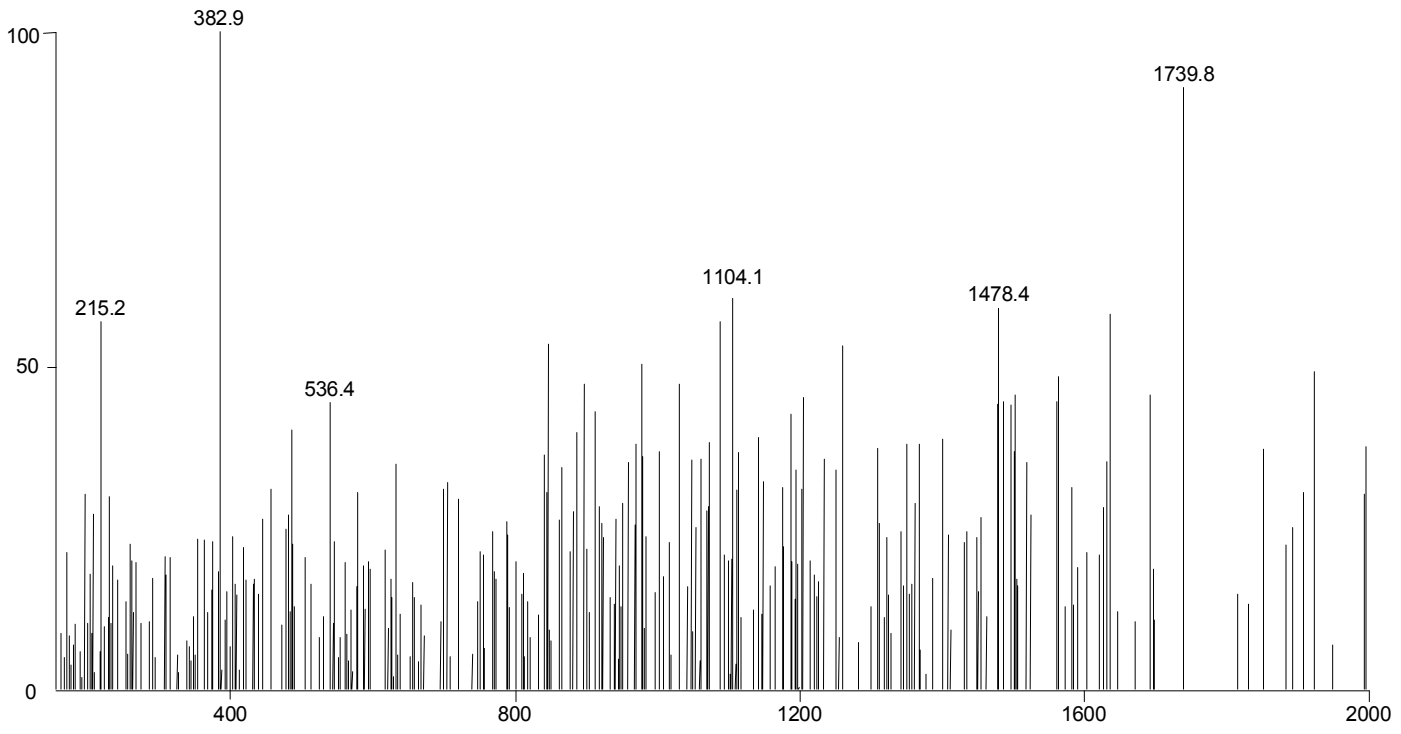

Figure 5.2-Spectrum of unseparated fungal exudate performed in positive ion mode. Flow rate, $0.10 \mu \mathrm{L} / \mathrm{min}$. Ionization voltage, $1.4 \mathrm{kV}$ 


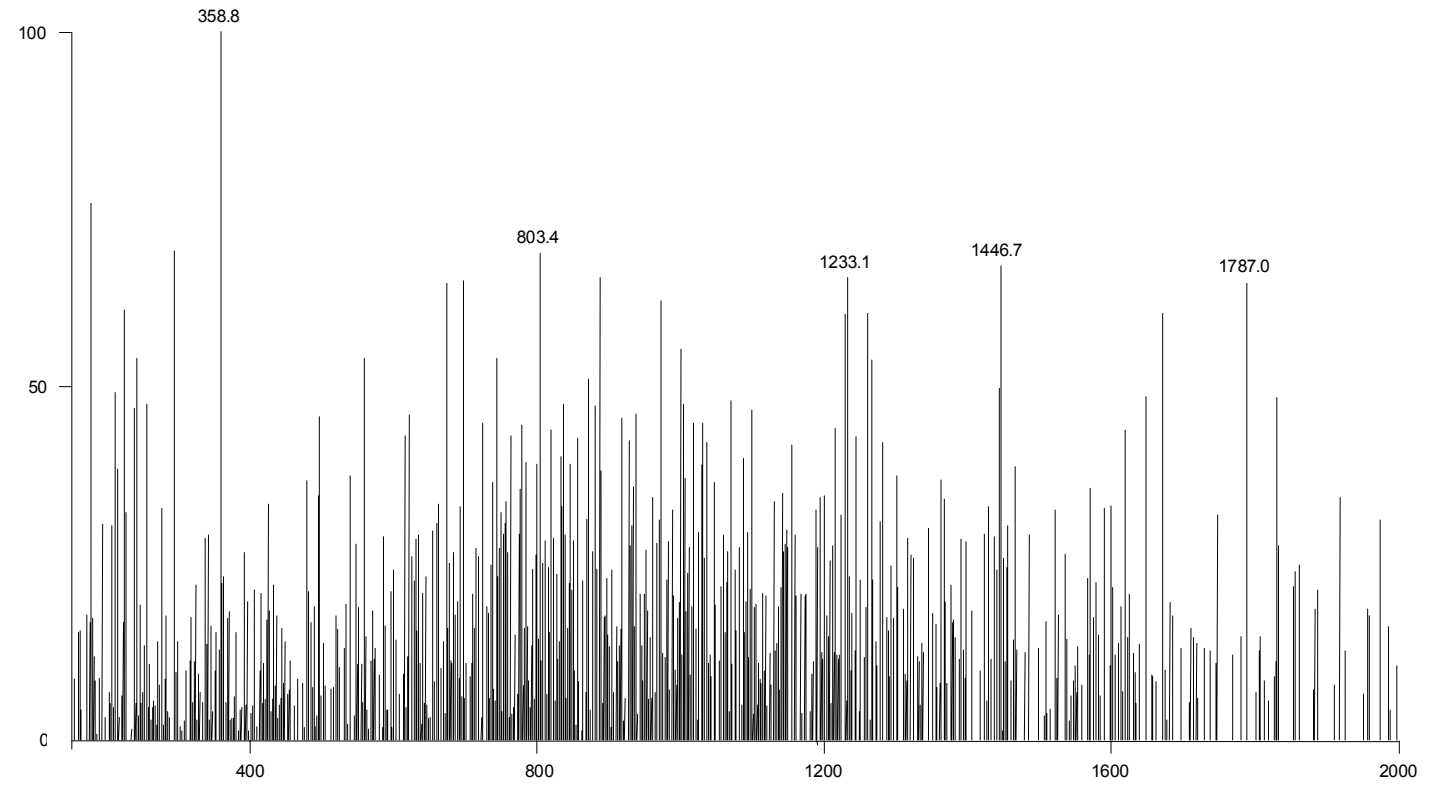

Figure 5.3-Spectrum of unseparated fungal exudate performed in negative ion mode. Flow rate, $0.10 \mu \mathrm{L} / \mathrm{min}$. Ionization voltage, $1.4 \mathrm{kV}$

Spectra that resulted from IMAC isolated samples were found to be less complex. Figure 5.4 shows a representative spectrum of IMAC purified pigments analyzed in negative ion mode. This data shows more distinct ion signals than those found in Figures 5.2-5.3. Furthermore, the signals that are observed are somewhat more stable than any found in the bulk exudate spectra. This observation is also seen in positive ion mode analysis (Figure 5.5). However, in both ionization modes, the background is too high to readily and confidently assess these signals. The noise masks the isotopic distribution that can be used to calculate the charge state. 


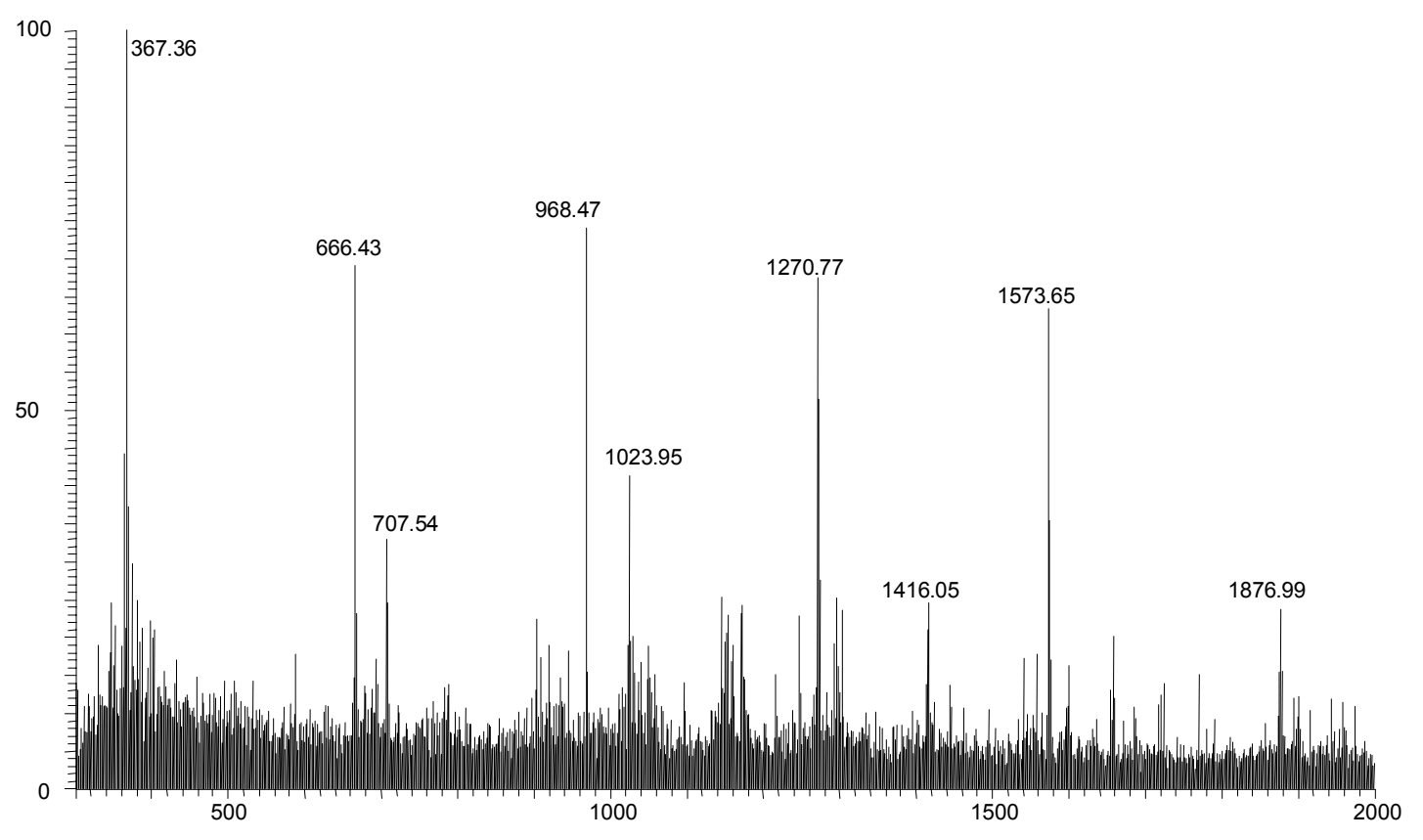

Figure 5.4-Spectrum of IMAC isolated exudate performed in negative ion mode. Flow rate, $0.10 \mu \mathrm{L} / \mathrm{min}$. Ionization voltage, $1.4 \mathrm{kV}$

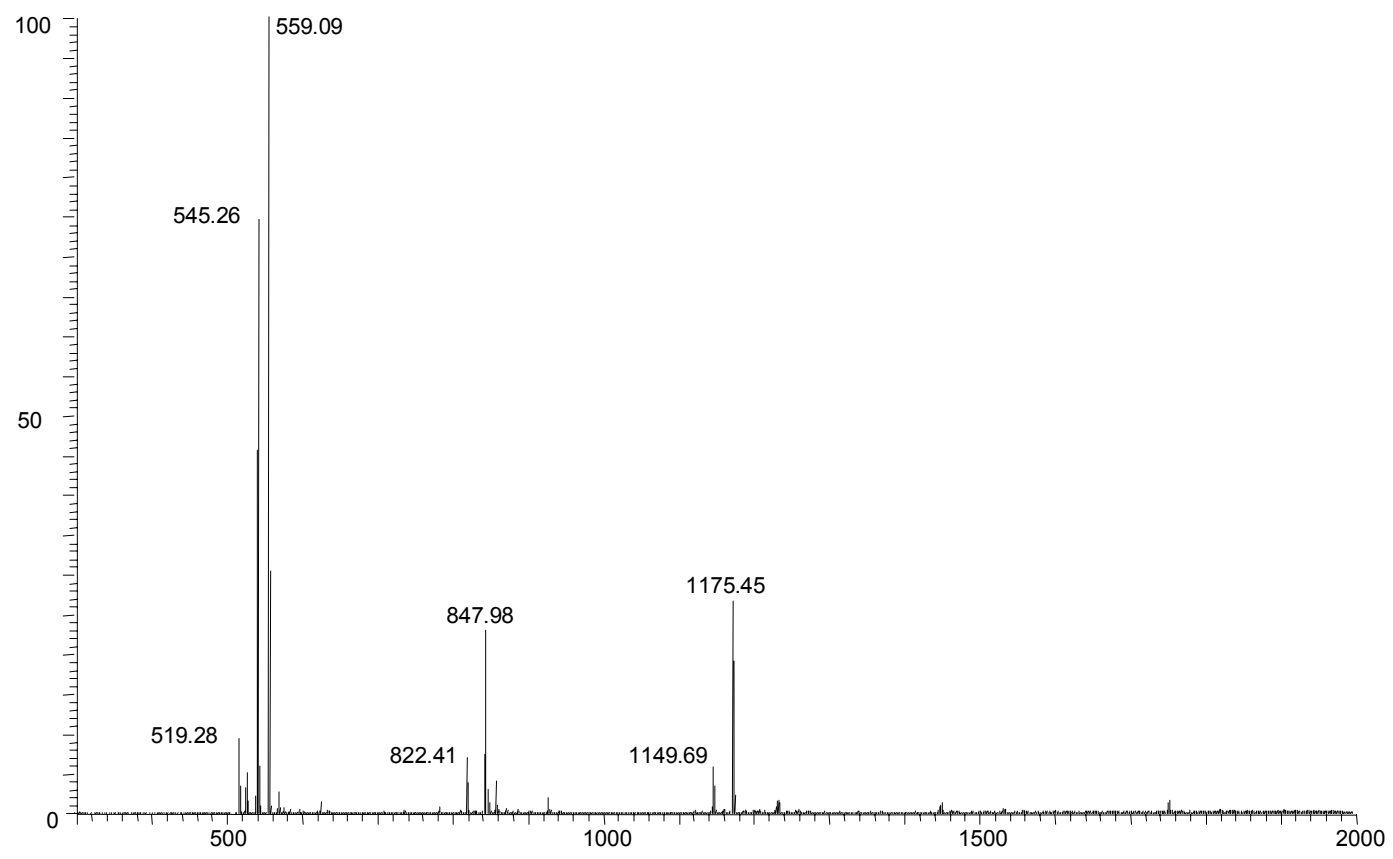

Figure 5.5-Spectrum of IMAC isolated exudate performed in positive ion mode. Flow rate, $0.10 \mu \mathrm{L} / \mathrm{min}$. Ionization voltage, $1.4 \mathrm{kV}$ 


\subsubsection{Removal of free $\mathrm{Al}^{3+}$ from the IMAC eluent with RP extraction}

Direct analysis of the IMAC extracted eluent proved to be unsuccessful. However, it was not directly apparent why the analysis failed and it was noted that it could be for at least one of two reasons. One possible reason was even after IMAC extraction the pigment exudate sample might be too complex for analysis. If there are too many exudate ligands present in the IMAC eluent solution, then a multi-dimensional separation might be required to reduce the sample complexity to levels appropriate of the ion-trap MS. Another cause could be the presence of $\mathrm{Al}^{3+}$ in the eluent mixture. If $\mathrm{Al}^{3+}$ was present during the ESI-MS analysis and multiple ligands complex an $\mathrm{Al}^{3+}$, then the presence of $\mathrm{Al}^{3+}$ will increase the number of species present and the complexity. Therefore, it is critical to investigate the fate of the $\mathrm{Al}^{3+}$ in the IMAC eluent solution, to determine if it is free in solution or if is

complexed by the ligands. A RP extraction method was used to remove the free $\mathrm{Al}^{3+}$ prior to MS analysis as described in Section 4.

Spectra obtained from the analysis of IMAC-RP purified samples showed a significant increase in signal stability and resolution. Therefore, removal of $\mathrm{Al}^{3+}$ reduced the spectral complexity and increased the stability of the ion signals. Additionally, there was a decrease in observed background signal. Figure 5.6 contains a spectrum collected in the negative ion mode following IMAC-RP purification. A well-defined group of signals are maintained during MS analysis, demonstrating the effectiveness of the purification steps in providing a clean sample for further data analysis.

\subsubsection{Assaying for $\mathrm{Al}^{3+}$ Complexation}

ICP-AES analysis of RP fractions showed trace levels of $\mathrm{Al}^{3+}$ along with the pigments. It was not known, however, whether this level was significant since the moles of 
pigment removed from the column was also unknown. By changing the $\mathrm{pH}$ and adding $\mathrm{Al}^{3+}$ in the IMAC-RP purified samples, the ability of isolated and purified pigment exudates to bind $\mathrm{Al}^{3+}$ was investigated. The samples analyzed by MS for this study were all IMAC-RP purified and treated as described in section 5.2.3. The $\mathrm{pH}$ was adjusted and $\mathrm{Al}^{3+}$ was added to determine if binding of $\mathrm{Al}^{3+}$ could be observed by observing ion signal shifts. Signal shifts between untreated IMAC-RP purified samples and those that were adjusted would confirm that the $\mathrm{Al}^{3+}$ was removed by the $\mathrm{RP}$ purification at $\mathrm{pH} 11$. The ICP-AES data suggests the pigments will not bind $\mathrm{Al}^{3+}$ at $\mathrm{pH} 11$ because the data shows most $\mathrm{Al}^{3+}$ is free in solution at this $\mathrm{pH}$. Upon lowering the $\mathrm{pH}$, a change in expressed signals due to both protonation and binding of $\mathrm{Al}^{3+}$ from solution is expected. However, if the $\mathrm{Al}^{3+}$ and exudates are complexed after IMAC purification, no signal shifts will be observed.

After the acidification and $\mathrm{Al}^{3+}$ addition, spectra of the exudates gathered from the ion trap show a noticeable difference among the different samples. This shift in ion signal upon the addition of $\mathrm{Al}^{3+}$ indicates that the exudates are unbound after IMAC-RP purification. If the mass spectra contained the same ion signals before and after the addition of $\mathrm{Al}^{3+}$, it would signify the exudates are already bound, but such is not the case. Figure 5.6 contains a representative mass spectrum of the IMAC-RP purified sample that was untreated in negative ion mode. It is expected that ion detection would be greater in negative ion mode at $\mathrm{pH} 11$ for an unbound acidic molecule. As can be observed in Figure 5.6, a number of distinct peaks are present, indicating the presence of a variety of molecules in the heterogeneous sample. However, when this $\mathrm{pH} 11$ IMAC-RP fraction is analyzed in positive ion mode, the only resolved signal present is due to matrix components 
implying there are no positive pigments present at $\mathrm{pH} 11$ (not shown). When $0.1 \mathrm{ppm} \mathrm{Al}^{3+}$ is present in the IMAC-RP sample, the negative ion mode spectra noticeably changes. Figure 5.7 is the spectra of the $\mathrm{pH} 11$ IMAC-RP sample containing $0.1 \mathrm{ppm} \mathrm{Al}^{3+}$ in negative ion mode and contains none of the dominant ion signals of the ion signals found in Figure 5.6.

Previously, our IMAC data presented in Chapter 3 indicated that exudates bind $\mathrm{Al}^{3+}$ strongly at $\mathrm{pH} 3$ and are likely to be uncomplexed at $\mathrm{pH} 11$. Therefore, if $\mathrm{Al}^{3+}$ was indeed removed as intended by the $\mathrm{RP}$ extraction, addition of $\mathrm{Al}^{3+}$ and lowering of the $\mathrm{pH}$ to 3 of the IMAC-RP purified sample would promote the complexing of the newly added $\mathrm{Al}^{3+}$ and exudates. If ion signal shifts do occur after the addition of $\mathrm{Al}^{3+}$ to the sample at $\mathrm{pH} 3$, the complexation of exudates and $\mathrm{Al}^{3+}$ in solution is confirmed and it can be reasoned the exudate was uncomplexed prior to acidification. If no ion signal shift is observed, it is shown that the fungal exudates can no longer bind $\mathrm{Al}^{3+}$, and the most likely explanation for this behavior was that the $\mathrm{Al}^{3+}$ was not actually removed by the attempted RP purification at $\mathrm{pH}$ 11. Figure 5.8 contains a spectrum of the IMAC-RP sample after being adjusted to pH 3 analyzed in positive ion mode. As would be anticipated, discrete ion signals are present in positive ion mode at lower $\mathrm{pH}$. Furthermore, analysis of the $\mathrm{pH} 3$ purified exudates in negative ion mode showed no stable signals at $\mathrm{pH} 3$, suggesting all acidic sites are protonated (not shown). Once the IMAC-RP sample at $\mathrm{pH} 3$ was put in the presence of $0.1 \mathrm{ppm} \mathrm{Al}\left(\mathrm{NO}_{3}\right)_{3}$ and analyzed in positive ion mode, there is a change in the signals observed in the mass spectrum as represented in Figure 5.9. Because all other conditions are kept the same except the presence of $\mathrm{Al}^{3+}$, these shifts suggest a variety of binding orientations and complexes are formed upon the addition of $\mathrm{Al}^{3+}$. Therefore, the observed 
change in signal with the addition of $\mathrm{Al}^{3+}$ to IMAC-RP purified samples at low $\mathrm{pH}$, along with previously noted ICP-AES data, points towards the elution of $\mathrm{Al}^{3+}$ and exudates as uncomplexed ions during IMAC isolation and elution.

It is not apparent why the $\mathrm{pH} 11$ negative ion spectra show a large change in the ion signals upon addition of the $\mathrm{Al}^{3+}$, which are indicative of $\mathrm{Al}^{3+}$ binding. In bulk solution, binding of $\mathrm{Al}^{3+}$ by the exudate pigments was not observed. A possible explanation for the observed binding in the ESI-MS analyzed samples is reactions that take place within the Taylor cone. As desolvation occurs, ions are forced closer in space, and therefore can potentially undergo reactions that would not typically occur under standard conditions [25]. As the sample droplets dry, volatile liquids such as the $\mathrm{NH}_{4} \mathrm{OH}$ are driven off, which can change the $\mathrm{pH}$ and increase the concentration of the $\mathrm{Al}^{3+}$ and pigment exudates as the droplet volume decreases during desolvation. Both the $\mathrm{pH}$ change and increase in concentration increase the probability of binding. These binding reactions would lead to spectra that do not depict the true structure of the unbound exudates. This behavior would explain why pigments appear to bind $\mathrm{Al}^{3+}$ at higher $\mathrm{pH}$ during ESI-MS analysis. Furthermore, a clean sample is needed as a control for comparison with spectra of aliquots that are spiked with $\mathrm{Al}\left(\mathrm{NO}_{3}\right)_{3}$. The success of RP extractions and purification performed offline before MS analysis proved very effective at cleaning up the samples. The time required to perform an online HPLC separation was not viable due to the formation of crystals on the ESI tip. Aliquots from low pressure RP separations, however, could be prepared in bulk and analyzed under several conditions and/or times. 


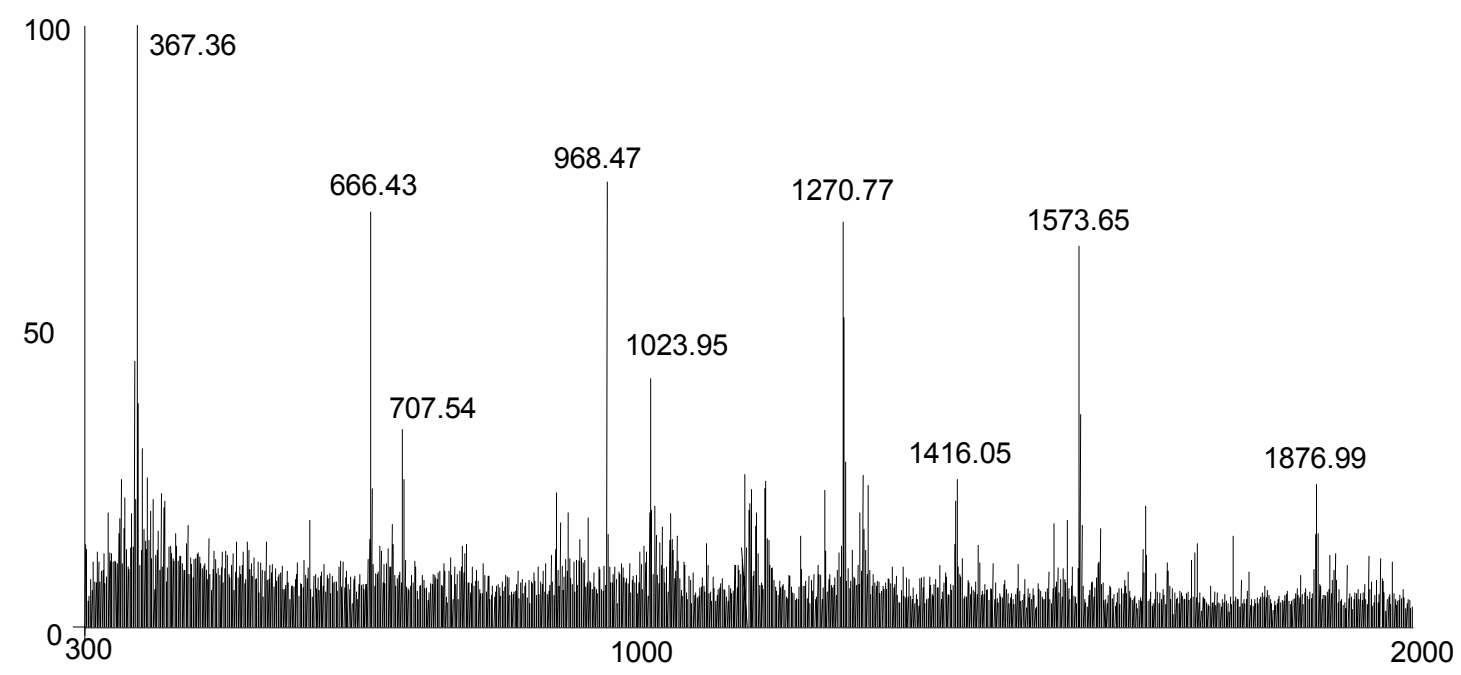

Figure 5.6-Mass spectrum of post IMAC-RP sample at $\mathrm{pH} 11$ with no $\mathrm{Al}^{3+}$ in negative ion mode.

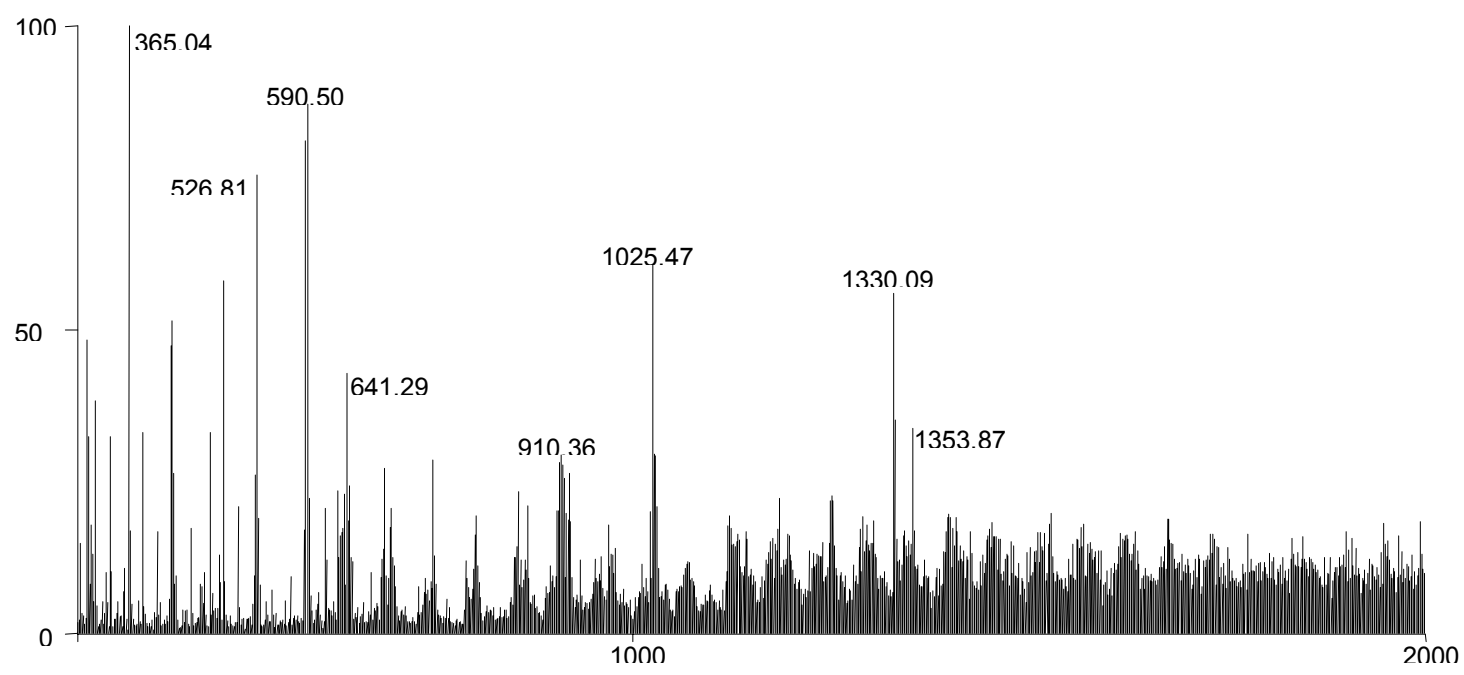

Figure 5.7-Mass spectrum of post IMAC-RP sample at $\mathrm{pH} 11$ with $0.1 \mathrm{ppm} \mathrm{Al}^{3+}$ in negative ion mode. 


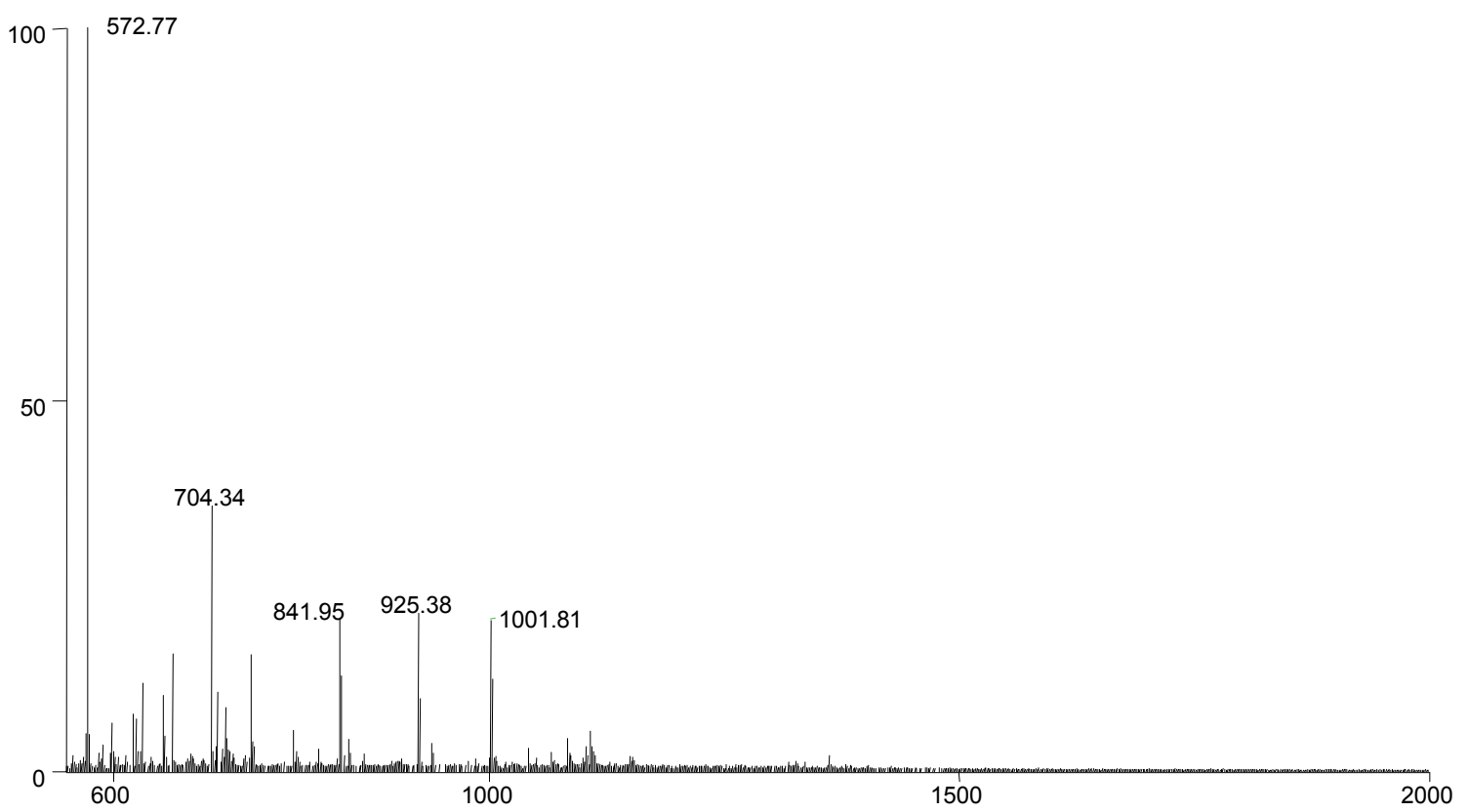

Figure 5.8-Mass spectrum of post IMAC-RP sample at $\mathrm{pH} 3$ with no $\mathrm{Al}^{3+}$ in positive ion mode.

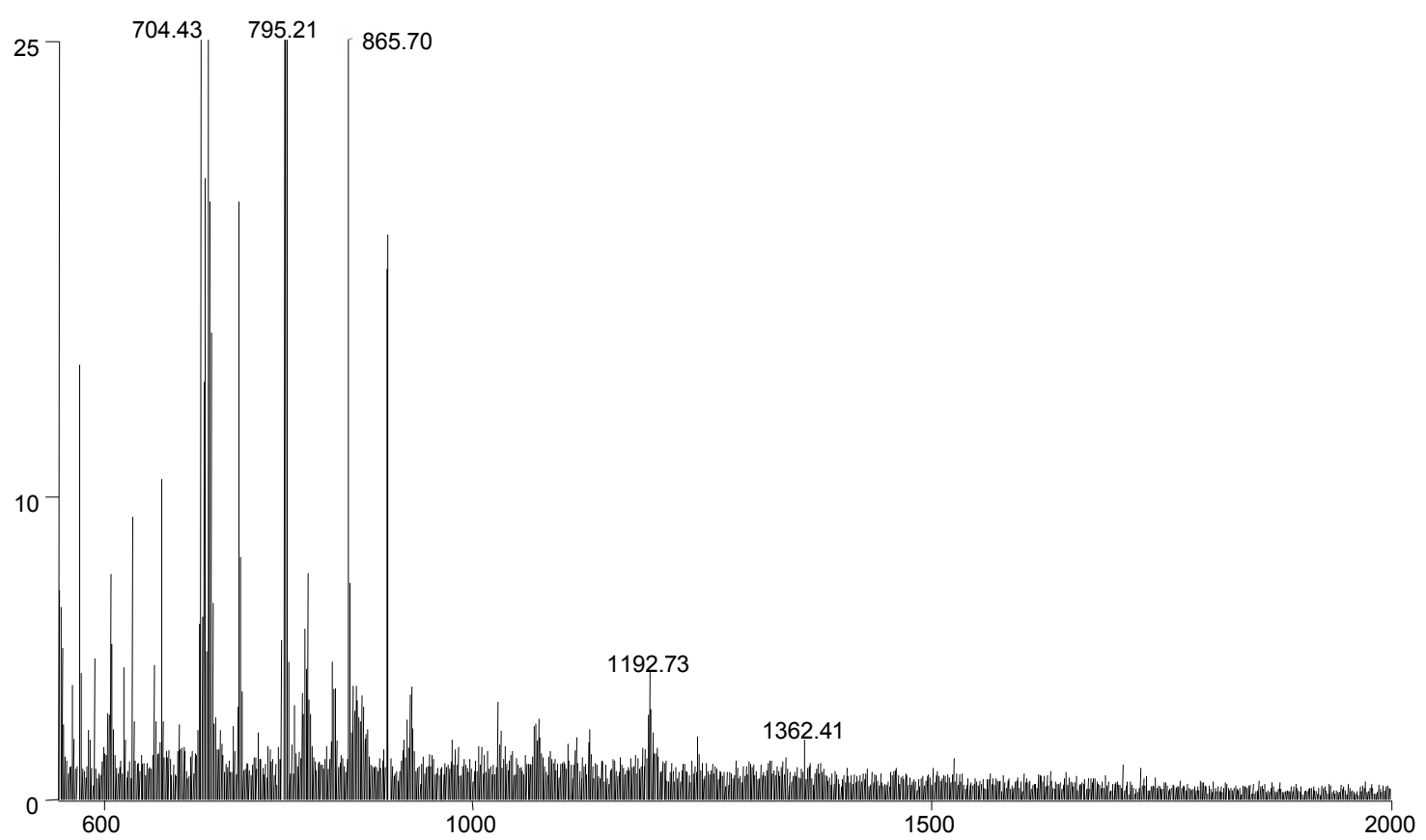

Figure 5.9-Mass spectrum of post IMAC-RP sample at $\mathrm{pH} 3$ with $0.1 \mathrm{ppm} \mathrm{Al}^{3+}$ in positive ion mode. 


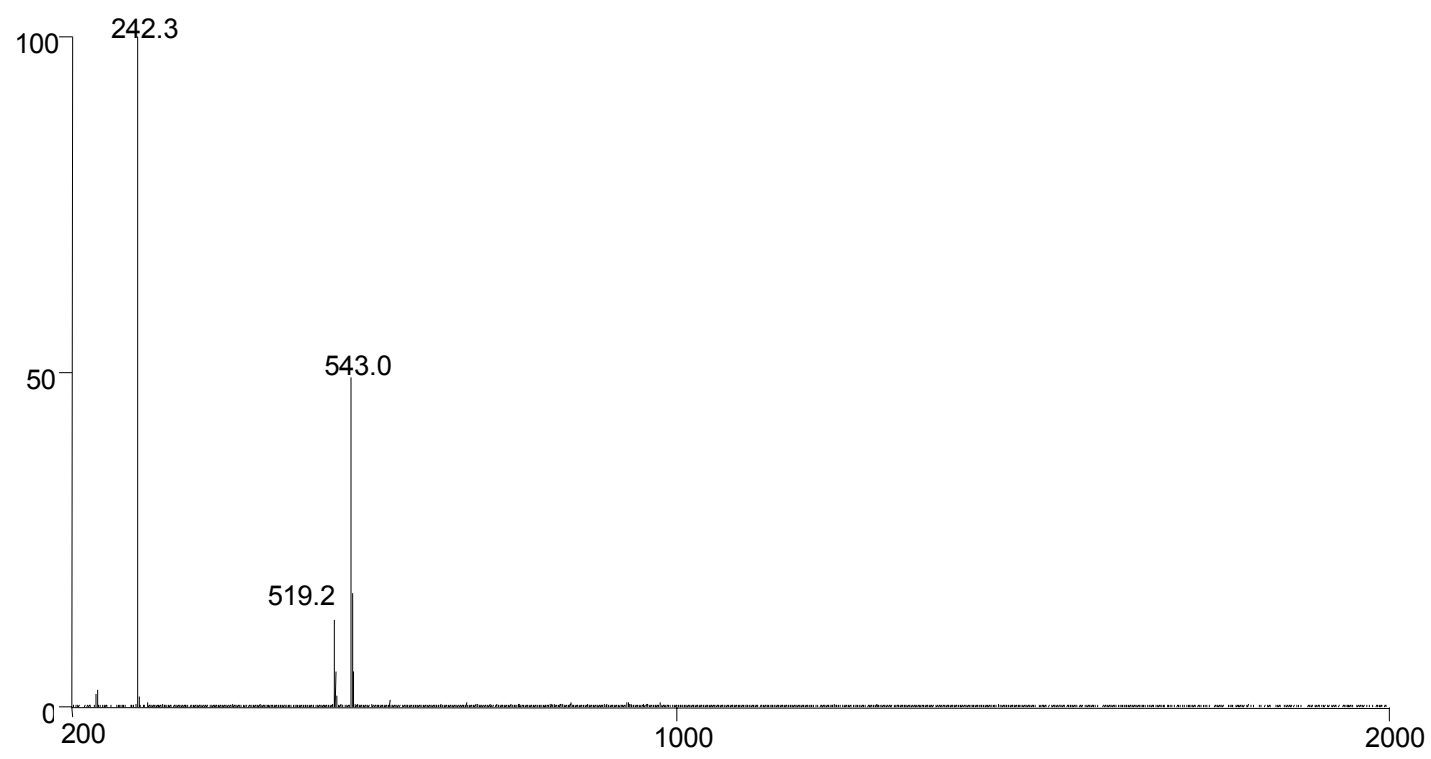

Figure 5.10-Mass spectrum of background performed in positive ion mode. Solution contains $1 \%$ ammonium hydroxide, $0.05 \%$ tetrabutylammonium hydroxide, and $90 \%$ acetonitrile.

\subsection{Conclusions}

The MS data shows that these fungal exudates are quite complex and require some form of isolation prior to qualitative analysis. Using IMAC and RP sequentially, the $\mathrm{Al}^{3+}$ binding fungal exudates were purified and successfully prepared for MS analysis. These purification steps successfully isolated the exudates of interest and removed $\mathrm{Al}^{3+}$ from the matrix prior to MS analysis.

IMAC and RP purifications performed in series provide sufficiently clean samples for MS analysis. With the $\mathrm{Al}^{3+}$-nonspecific compounds and free $\mathrm{Al}^{3+}$ removed, discrete ion signals are observed. The assays in which $\mathrm{Al}^{3+}$ was added back to the solution, confirm the free $\mathrm{Al}^{3+}$ is removed by the by $\mathrm{RP}$-extraction. This $\mathrm{Al}^{3+}$ add back assay corroborates the ICP-AES analysis of the RP fractions that the $\mathrm{Al}^{3+}$ was removed, Addition evidence of the removal of $\mathrm{Al}^{3+}$ was acquired, because one could not be certain that the trace levels of $\mathrm{Al}^{3+}$ were found in the RP fractions containing the fungal exudates was insignificant because the 
moles of fungal exudate present was also unknown. Additionally, these spectra show the heterogeneous nature of the exudate mixture due to the number of unrelated peaks present. 


\section{Conclusions}

The fungal exudates utilized during the course of this research are complex and heterogeneous mixtures. The exudates are similar to humic acids in that they contain smaller organic acids and contain a variety of compounds in a given sample. Qualitative analysis by ESI-MS shows that the fungal exudates are a complex mixture of related molecules and illustrates the need for purification.

Although $\mathrm{Al}^{3+}$ IMAC has been previously reported in the literature, it has not been used for the isolation of molecules with such high binding strength at low $\mathrm{pH}$. IDA columns are known to bind $\mathrm{Al}^{3+}$, but, such as the binding capacity of IDA resins are not available. Through the span of this research, an optimized technique for isolating $\mathrm{Al}^{3+}$ and $\mathrm{Al}^{3+}$ specific ligands was successfully devised. The key component that permitted the successful elution of exudates was the use of $\mathrm{NH}_{4} \mathrm{OH}$ as the elutor. Because $\mathrm{Al}^{3+}$ is a very hard Lewis acid, it requires a hard Lewis base to dissociate the bond between the $\mathrm{Al}^{3+}$ and the immobilized IDA ligand. $\mathrm{NH}_{4} \mathrm{OH}$ meets this requirement because both $\mathrm{NH}_{3}$ and $\mathrm{OH}^{-}$ are hard Lewis bases. In fact, $\mathrm{NH}_{4} \mathrm{OH}$ also dissociates the $\mathrm{Al}^{3+}$ and exudates during IMAC elution. $\mathrm{NH}_{4} \mathrm{OH}$ not only proved to be the most efficient elutor versus other attempted elutors, but is also volatile and imparts limited interference during UV-Vis quantitation.

Using RP separation followed by ICP-AES analysis, it was determined that $\mathrm{Al}^{3+}$ is free in solution and can be removed from the pigment mixture. At $\mathrm{pH} 11$, the exudates are fully retained on the RP-column, while the $\mathrm{Al}^{3+}$ is found to elute primarily in the void volume with trace amounts eluting in later fractions. In order to confirm that the trace levels of $\mathrm{Al}^{3+}$ found in the fungal exudate fractions were insignificant, an MS assay was developed to confirm that the fungal exudate ligands could bind $\mathrm{Al}^{3+}$ after purification. 
After the isolation of strongest $\mathrm{Al}^{3+}$-binding fraction of fungal exudates and removal of $\mathrm{Al}^{3+}$ from the matrix, discrete ion signals were obtained using ESI-MS. Initial attempts at ESI-MS analysis of the unseparated and poorly purified fungal exudate mixtures yielded complex spectra that resembled ESI-background. During negative ion mode analysis of the IMAC-RP purified sample at $\mathrm{pH} \mathrm{11,} \mathrm{the} \mathrm{signals} \mathrm{correspond} \mathrm{to} \mathrm{the}$ uncomplexed ligands in solution. After adjusting the sample $\mathrm{pH}$ to 3 and adding $\mathrm{Al}^{3+}$ to the sample, the observed signals relate to the $\mathrm{Al}^{3+}$-exudate complex. Because the adjustment of sample $\mathrm{pH}$ and addition of $\mathrm{Al}^{3+}$ to the sample changes the signals observed, it is determined that the ligands are unbound after IMAC elution and rebind $\mathrm{Al}^{3+}$ at $\mathrm{pH} 3$.

It is anticipated that these methods will provide means to isolate naturally occurring ligands from the environment, not just those produced in a lab setting. Furthermore, broadening the scope from $\mathrm{Al}^{3+}$ to other metals is also expected. With the ability to isolate, separate, and qualitatively analyze samples that are responsible for metal binding in the environment; details regarding the nature of such compounds can more easily be obtained. 


\section{REFERENCES}

[1] J.L. Halvin, et al., Soil Fertility and Fertilizers (1999)

[2] L.V. Kochian, Annu. Rev. Plant Physiol. Plant Mol. Biol. 46 (1995) 237

[3] H.E. Matsumoto, M. Sasaki, Biotechnology and Biodiversity in Agriculture/Forestry (1999)

[4] M. Barinaga, Science 276 (1997) 1497

[5] E. Delhaize, P.R. Ryan, Plant Physiology 107 (1995) 315

[6] J.F. Ma, Plant and Cell Physiology 41 (2000) 383

[7] H. Marshner (1995) Mineral Nutrition of Higher Plants. Academic Press. NY.

[8] S.E. Smith, D.J. Read (1997) Mycorrhizal Symbiosis. Academic Press. NY.

[9] S.F. Wright, A. Upadhyaya. Plant Soil 198 (1998) 97

[10] J.R. Cumming, et al., Unpublished data

[11] J. Porath, Protein Expression and Purification 3 (1992) 263

[12] P.E. Dykema, P.R. Sipes, Plant Molecular Biology 41 (1999) 139

[13] J. Porath, B. Olin Biochemistry 22 (1983) 1621

[14] K.N. Saito, Akira Polyhedron 9 (1990) 215

[15] G.J. Taylor, Soil Sci. Plant Anal. 19 (1988) 1179

[16] J.F. Ma, P.R. Ryan, E. Delhaize. TRENDS in Plant Science 6 (2001), 273

[17] A.A. Meharg, J.W.G. Cairney, Advances in Ecological Research 30 (2000) 69

[18] G. Jentschke, D.L. Godbold, Physiol. Plant 109 (2000) 107

[19] Urmila Basu et al. Physiologia Plantarum 106 (1999) 53

[20] Gary L. Miessler, Donald A. Tarr Inorganic Chemistry $2^{\text {nd }}$ ed. Prentice-Hall Inc. (1999)

[21] E.K.M. Ueda, P.W. Gout, L. Morganti Journal of Chromatography A, 988 (2003) 1

[22] J. Porath Nature, 258 (1975) 598 
[23] Albert L. Lehninger, David L. Nelson, Michael Cox Principles of Biochemistry $2^{\text {nd }}$ ed. Worth Publishers (1993)

[24] C.F. Simpson Techniques in Liquid Chromatography Wiley Heyden Publications (1982)

[25] Edmond de Hoffmann, Vincent Stroobant Mass Spectrometry: Principles and Applications $2^{\text {nd }}$ ed. Wiley Publishing (2002)

[26] Garrison Sposito The Environmental Chemistry of Aluminum CRC Press, Inc. (1989)

[27] B. Anupkumar, T.S. Rao, K.K. Satpathy Current Science, 80 (2001) 9

[28] Lyly Le, Chi, Kim Chi; Scott Tyldesley, Stephane Flibotte, Deborah L. Diamond, Michael A. Kuzyk, Marianne D. Sadar Clinical Chemistry 51 (2005) 695

[29] C. Baldwin, J. Cumming, A. Timperman, Analyst, 130 (2005) 318

[30] D.H. Marx, J.D. Artman, Reclamation Review, 2 (1979) 23

[31] D.A. Wilkins, Agriculture, Ecosystems, \& Environment, 35 (1991) 245

[32] Thomas E. Furia, CRC Handbook of Food Additives, $2^{\text {nd }}$ ed. CRC Press, Inc. (1972) 